\title{
Renormalized Lindblad driving: A numerically exact nonequilibrium quantum impurity solver
}

\author{
Matan Lotem $\odot,{ }^{1}$ Andreas Weichselbaum $\odot,{ }^{2,3}$ Jan von Delft $\odot,{ }^{3}$ and Moshe Goldstein $\odot^{1}$ \\ ${ }^{1}$ Raymond and Beverly Sackler School of Physics and Astronomy, Tel Aviv University, Tel Aviv 6997801, Israel \\ ${ }^{2}$ Department of Condensed Matter Physics and Materials Science, Brookhaven National Laboratory, Upton, New York 11973-5000, USA \\ ${ }^{3}$ Arnold Sommerfeld Center for Theoretical Physics, Center for NanoScience, and Munich Center for Quantum Science and Technology, \\ Ludwig-Maximilians-Universität München, 80333 Munich, Germany
}

(Received 22 April 2020; revised 16 August 2020; accepted 19 August 2020; published 9 October 2020)

\begin{abstract}
The accurate characterization of nonequilibrium strongly correlated quantum systems has been a longstanding challenge in many-body physics. Notable among them are quantum impurity models, which appear in various nanoelectronic and quantum computing applications. Despite their seeming simplicity, they feature correlated phenomena, including small emergent energy scales and non-Fermi-liquid physics, requiring renormalization group treatment. This has typically been at odds with the description of their nonequilibrium steady state under finite bias, which exposes their nature as open quantum systems. We present a numerically exact method for obtaining the nonequilibrium state of a general quantum impurity coupled to metallic leads at arbitrary voltage or temperature bias, which we call "RL-NESS" (renormalized Lindblad-driven nonequilibrium steady state). It is based on coherently coupling the impurity to discretized leads which are treated exactly. These leads are furthermore weakly coupled to reservoirs described by Lindblad dynamics which impose voltage or temperature bias. Going beyond previous attempts, we exploit a hybrid discretization scheme for the leads together with Wilson's numerical renormalization group, in order to probe exponentially small energy scales. The steady state is then found by evolving a matrix-product density operator via real-time Lindblad dynamics, employing a dissipative generalization of the time-dependent density matrix renormalization group. In the long-time limit, this procedure successfully converges to the steady state at finite bond dimension due to the introduced dissipation, which bounds the growth of entanglement. We thoroughly test the method against the exact solution of the noninteracting resonant level model. We then demonstrate its power using an interacting two-level model, for which it correctly reproduces the known limits, and gives the full $I-V$ curve between them.
\end{abstract}

DOI: 10.1103/PhysRevResearch.2.043052

\section{INTRODUCTION}

Quantum impurity models have fascinated theoreticians for several decades. These models seem extremely simple: they describe a small, typically interacting, quantum system, i.e., the impurity, coupled to a noninteracting environment. The quantum impurity consists of only a few degrees of freedom, so that its spectrum can be obtained exactly. However, once this interacting impurity is coupled to the seemingly innocent quadratic environment, it gives rise to highly correlated behavior and exotic phenomena which cannot be explained solely in terms of the bare impurity, such as the Kondo effect (including its non-Fermi-liquid multichannel varieties) [1,2]. Quantum impurities can thus be seen as the basic building blocks of higher-dimensional strongly interacting systems. The most striking feature of these arising phenomena is that they can occur at emergent energy scales which, a priori, do not appear in the Hamiltonian of either the bare impurity or the

Published by the American Physical Society under the terms of the Creative Commons Attribution 4.0 International license. Further distribution of this work must maintain attribution to the author(s) and the published article's title, journal citation, and DOI. environment. An example is the Kondo temperature, which can be smaller by several orders of magnitude than any bare energy scale. Thus, in order to expose the physics of these models, they must be analyzed in a renormalization group (RG) framework. As of today, the most successful method for treating such problems, in or close to equilibrium, is Wilson's numerical renormalization group (NRG) [3,4], a numerically exact RG procedure for integrating out high-energy modes and probing arbitrarily small energy scales.

A wide range of devices with various nanoelectronic and quantum computing applications, including semiconductor quantum dots [5,6], carbon nanotubes coupled to metallic leads $[7,8]$, and molecular junctions $[9,10]$, can be described as quantum impurity models, with the environment corresponding to two macroscopic leads. Most of their applications involve imposing a voltage (chemical potential) or temperature bias between the leads which results in a nonequilibrium steady state (NESS), with a tunneling current flowing through the impurity. Experimental results for such systems have successfully been explained in different limits, e.g., by linear response theory together with equilibrium NRG for small bias, or by solving a master equation at large temperature or voltage bias [11]. However, for arbitrary bias, a quantitative theoretical description of the NESS properties is still an open challenge. Any complete solution to this problem must 
(a)

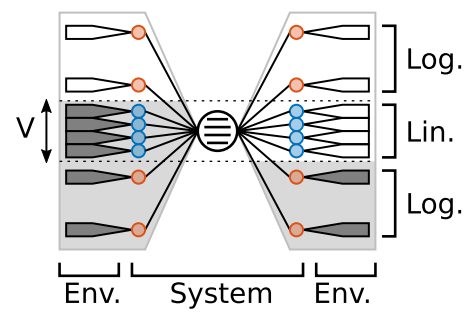

(b)

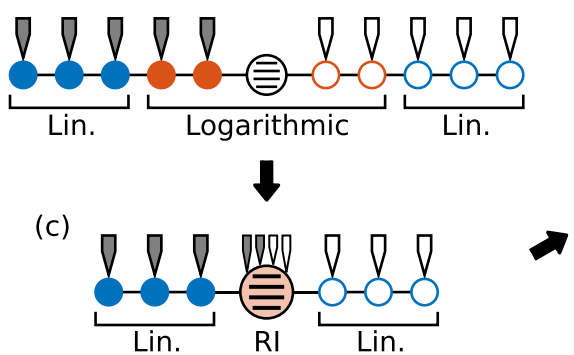

(d)

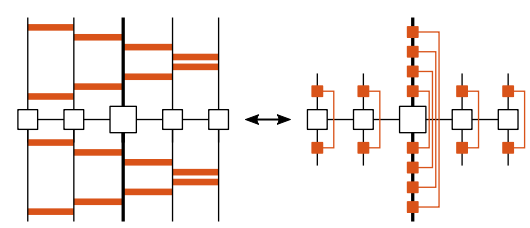

FIG. 1. Overview of the RL-NESS method (for more details see Figs. 2 and 3). (a) Two leads with continuous spectra and at a temperature or voltage bias are replaced by a discrete set of energy levels coupled to Lindblad baths. This discrete set is chosen by logarithmic discretization (red) above the bias scale and linear discretization below it (blue). (b) The system is mapped to a tight-binding chain with a local Lindblad bath coupled to each site. (c) High-energy modes are integrated out by equilibrium NRG on the logarithmic sector, resulting in a renormalized impurity (RI). (d) The system is evolved in real time toward the long-time limit steady state, using a dissipative variant of tDMRG for a matrix-product density operator.

(i) capture interaction induced many-body correlations, (ii) resolve a wide range of energy scales, and (iii) deal with an open system at its steady state.

Attempts to generally tackle this problem analytically, e.g., in an RG framework [12-14] or by Keldysh field integral formulation [15], are so far restricted to uncontrolled approximations. Bethe ansatz approaches have also been tried [16,17], but are typically case specific. Therefore, much focus has been placed on finding a general numerical solution. A class of such attempts is based on capturing the many-body correlations by modeling the environment as large (but finite) leads, and evolving the many-body state of this finite system toward a finite-time quasi-steady state, e.g., using the time-dependent density matrix renormalization group (tDMRG) method [18-20]. This approach has further been extended by treating the finite leads as open systems, governed by Lindblad dynamics, and similarly evolving in time toward a well-defined steady state [21-23]. The Lindblad approach has also been recently investigated in the context of density functional theory [24]. However, these attempts are typically limited in terms of the range of energy scales explored by the finite number of energy levels in the leads, with no RG procedure exploited in order to integrate out high-energy modes. Other numerically exact approaches applied to this problem are reported in [25-28], but are also not designed to explore the wide range of energy scales. Two attempts to leverage the unrivaled success of NRG in equilibrium and extend it out of equilibrium are the so-called scattering-states NRG [29] and the NRG-tDMRG scheme [30], with the latter a predecessor of the method presented in this paper. These attempts have been quite successful at resolving a wide range of energy scales, while also capturing the many-body correlations. However, the former is plagued by logarithmic discretization artifacts within the dynamical energy window, while the latter is based on nondissipative time evolution of a finite, and thus closed, system, which results in a quasi-steady state in a limited time interval, making it challenging to extract steady-state observables.

In this work we present an algorithm combining the full power of NRG and tDMRG for capturing many-body correlations at a wide range of energy scales, together with open system dynamics, in order to obtain an actual nonequilibrium steady state. In what follows, we will refer to this approach as the renormalized Lindblad-driven NESS (RL-NESS) method.
Our starting point is a general impurity coupled to continuous leads (i.e., leads with a continuous spectrum). As shown in Fig. 1(a), each continuous lead is separated into a finite set of representative discrete energy levels, which in turn are coupled to the remaining continuous modes. The impurity together with this finite set of energy levels (large enough to allow the coherent formation of, e.g., the Kondo screening cloud, and the emergence of energy scales such as the Kondo temperature), is considered as an open system, coupled to an environment consisting of the remaining continuous modes, which are traced out. The latter is performed under the Born and Markov approximations, i.e., that the environment is memoryless and indifferent to the state of the system. As a result, the dynamics of the system is governed by a Lindblad [31] master equation:

$$
\frac{d \rho}{d t}=\mathcal{L} \rho=-i[H, \rho]+\mathcal{D} \rho .
$$

The Liouvillian superoperator $\mathcal{L}$ can be separated into a von Neumann term consisting of the discrete system Hamiltonian $H$, and a dissipative superoperator $\mathcal{D}$, describing a suitably modeled dissipation into the environment. The two key elements of our method are (i) the specific choice of discrete energy levels, such that high-energy modes can be integrated out, and (ii) the numerical solution of the Lindblad equation in the low-energy dynamical regime, formulated as a tensornetwork algorithm.

With these requirements in mind, the Lindblad equation is obtained as follows: The Hamiltonian of the discrete system is derived by employing a mixed discretization scheme that crosses over from logarithmic to linear level spacing at the bias scale [30]. This permits integrating out modes whose energies are high compared to the bias voltage or temperature by means of NRG, with the logarithmic RG flow eventually cut off at this scale. Instead of formally deriving the dissipators, they are chosen based on two criteria: (i) the solution of the Lindblad equation reproduces the continuum limit, and (ii) Eq. (1) can be numerically solved efficiently. An important property of the chosen dissipators is that they are local in the basis in which the leads are diagonal. A set of exact transformations, dubbed the Lindblad-driven discretized leads (LDDL) scheme [32], is then applied to the Lindblad equation, mapping it to a so-called chain geometry, shown in Fig. 1(b), 
which, due the short rangedness (or locality) of interactions is more favorable for treatment in the tensor-network framework, e.g., by tDMRG [33,34]. At this stage, high-energy modes (far above the bias voltage and temperature scales) are integrated out using equilibrium NRG, arriving at a local Lindblad equation in an effective low-energy basis, as depicted in Fig. 1(c). The state of the system is represented as a matrix-product density operator (MPDO), and is evolved in real time by a dissipative variant of tDMRG in Liouvillian space, as shown in Fig. 1(d), until convergence to a steady state is obtained. Due to the dissipation induced by the environment, the entanglement entropy of the system saturates as a function of time, rather than diverging, as is the case in the absence of dissipation. Hence, the long-time limit steady state can be obtained with finite MPDO bond dimension. A full description of the method will be presented in Sec. II.

By repeating the simulation for different bias voltages, a full $I-V$ curve can be obtained. When numerically differentiated, one obtains the differential conductance. The method is demonstrated on two spinless fermionic models: the noninteracting resonant level model (RLM), and an interacting two-level model (I2LM). The RLM, discussed in Sec. III, can be solved exactly in the single-particle basis (in and out of equilibrium). It will therefore serve as a benchmark for the presented method. The I2LM, discussed in Sec. IV, contains two interacting dot levels with level spacing $\Delta$ and interaction energy $U$. Our method recovers known results in the limits of small and large bias, yet goes beyond them by giving the full $I-V$ curve. Conclusions and future directions are discussed in Sec. V, followed by a series of appendices covering technical details.

\section{METHOD}

In this section the RL-NESS method is outlined in detail. The initial part follows much of the strategy in Ref. [30]. We start by presenting a general impurity model with continuous leads in Sec. II A. The leads are then discretized in Sec. II B, resulting in a Lindblad equation for a discrete system. In Sec. II C we follow by a short overview of the LDDL scheme, used to bring this equation into a local form, both in the Hamiltonian and in the dissipators. In Sec. II D we integrate out high-energy modes by NRG in order to obtain a renormalized impurity. In Sec. II E we describe a matrix-product density operator procedure for real-time evolution toward the steady state. Finally, in Sec. II F we discuss the extraction of observables from the obtained steady state. Steps II B-II D are described schematically in Fig. 2, and step IIE is described in Fig. 3. Throughout this section, superoperators acting on the density matrix will be represented in calligraphic script, while regular operators will be represented in roman script. Tensor-network calculations (NRG, MPDO evolution) were implemented using the QSPACE tensor library, which can exploit both Abelian and non-Abelian symmetries on a generic footing $[35,36]$.

\section{A. Model}

The total Hamiltonian of an impurity system can be generically separated into three parts: the (interacting) impurity, the noninteracting leads with a continuum density of states, and the coupling between them:

$$
H_{\text {total }}=H_{\text {dot }}+H_{\text {coupling }}+H_{\text {leads }} .
$$

The dot Hamiltonian describes $\lambda \in\{1, \ldots, m\}$ (here spinless) levels with onsite Coulomb repulsion $U$ :

$$
H_{\mathrm{dot}}=\sum_{\lambda=1}^{m} \varepsilon_{\lambda} n_{d \lambda}+\frac{U}{2} n_{d}\left(n_{d}-1\right),
$$

with fermionic creation operators $d_{\lambda}^{\dagger}$, and total impurity occupation $n_{d}=\sum_{\lambda} n_{d \lambda}$, where $n_{d \lambda}=d_{\lambda}^{\dagger} d_{\lambda}$. More complicated local interactions, such as exchange interactions or spin Hund's coupling, may also be incorporated.

The lead Hamiltonian in this work is described by two metallic, i.e., noninteracting, leads located left and right of the impurity. They are assumed to be featureless, with constant hybridization $\Gamma_{\alpha \lambda}$ of lead $\alpha \in\{L, R\}$ with impurity level $\lambda$ over a bandwidth $\varepsilon \in[-D,+D]$, resulting in the total hybridization strength $v_{\alpha \lambda} \equiv \sqrt{\frac{2 D \Gamma_{\alpha \lambda}}{\pi}}$. The lead and coupling Hamiltonians can therefore be written in the diagonal bath basis as

$$
\begin{gathered}
H_{\text {leads }}=\sum_{\alpha} \int_{-D}^{D} d \varepsilon \varepsilon c_{\alpha \varepsilon}^{\dagger} c_{\alpha \varepsilon}, \\
H_{\text {coupling }}=\sum_{\alpha \lambda} v_{\alpha \lambda} \underbrace{\int_{-D}^{D} \frac{d \varepsilon}{\sqrt{2 D}}\left(c_{\alpha \varepsilon}^{\dagger}\right.}_{\equiv c_{\alpha 0}^{\dagger}} d_{\lambda}+\text { H.c. }),
\end{gathered}
$$

where $c_{\alpha \varepsilon}^{\dagger}$ creates an electron in lead $\alpha$ at energy $\varepsilon$. As indicated, $c_{\alpha 0}^{\dagger}$ defines the normalized bath level that the impurity couples to, i.e., at the location of the impurity, obeying $\left\{c_{\alpha 0}, c_{\alpha 0}^{\dagger}\right\}=1$. The generalization to spinful and multichannel leads is straightforward, while the generalization to a featured hybridization function is conceptually also possible. Throughout, we assume the limit of large bandwidth, i.e., that all energy scales and parameters are much smaller than $D$. Without loss of generality then, the voltage bias is chosen symmetric with respect to the Fermi energy, so that the chemical potentials of the leads are $\mu_{L}=-\mu_{R}=-\frac{V}{2}$ (taking unit of charge $e=1$, throughout). For concreteness we will mostly concentrate on the case of zero temperature in both leads, but the described procedure also applies to finite and nonequal temperatures.

\section{B. Lindblad equation}

The Lindblad equation is a first-order linear differential equation. Its general solution, given some initial condition $\rho_{0}$, can thus be written by exponentiating the Liouvillian superoperator:

$$
\frac{\partial \rho}{\partial t}=\mathcal{L} \rho \Rightarrow \rho(t)=e^{\mathcal{L} t} \rho_{0} .
$$

The dynamics in our case is designed to have a unique nonequilibrium steady state defined by

$$
\mathcal{L} \rho_{\mathrm{NESS}} \equiv 0 \quad \Longleftrightarrow \quad \rho_{\mathrm{NESS}}=\lim _{t \rightarrow \infty} \rho(t),
$$


(a)
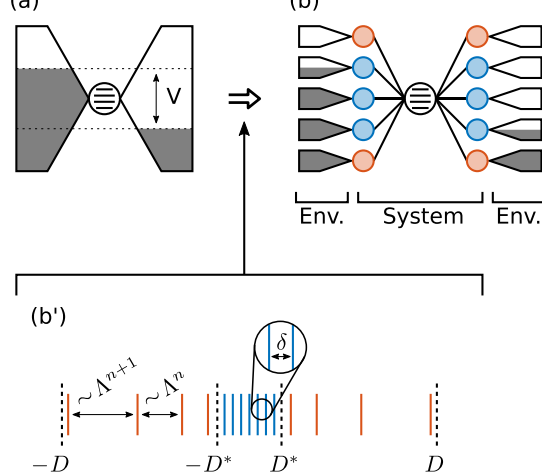

(c)

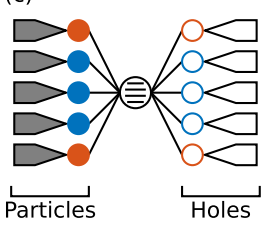

(d)
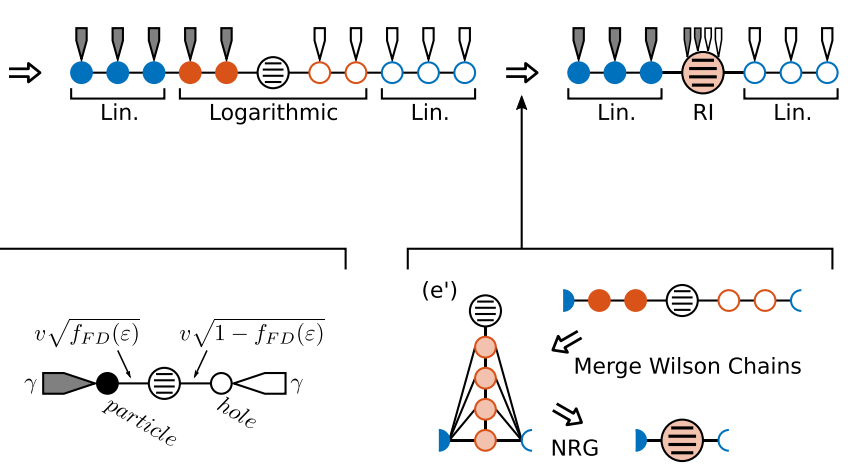

FIG. 2. Schematic description of the RL-NESS method leading up to the point of solving the Lindblad equation. (a) The system of interest is a general impurity coupled to two macroscopic and thus continuous leads at chemical potential difference $V$. This system can be mapped exactly onto (b), where the bath has been coarse grained into distinct intervals. Each of these is written as a representative level that the impurity couples to, and a continuous bath consisting of the remainder of the modes in that interval. The discrete set of energy levels together with the impurity thus form a finite system. The remaining lead modes are integrated out, resulting in a Lindblad bath coupled to each discrete energy level. $\left(b^{\prime}\right)$ The width of the intervals is chosen according to a logarithmic-linear discretization scheme, such that levels in the low-energy window $\left[-D^{*},+D^{*}\right]$ are equally spaced by $\delta$ (blue), with a smooth transition to logarithmic spacing $\sim \Lambda^{n}$ at large energies (red). (c) The targeted occupation of each individual lead mode, originally encoded in the couplings to the Lindblad baths in (b), can be transferred onto the lead-impurity couplings, such that the resulting two leads now represent particles or holes, and are driven to be completely full or empty, respectively. ( $\mathrm{c}^{\prime}$ ) This rotation of the local Liouvillian basis is performed separately for each original (physical) lead level. A general such level is coupled to the impurity with coupling constant $v$, and to two Lindblad baths, one filling it and the other emptying it, at rates proportional to $\gamma$ and chosen such that they drive the level toward its equilibrium occupation (determined by chemical potential and temperature). From the Liouvillian description, an auxiliary level is introduced at the same energy, and linear combinations of the two levels are chosen such that one is driven to be completely full (particle) and the other to be completely empty (hole), both at rate $\gamma$. (d) The particle and hole leads can be exactly mapped onto nearest-neighbor Wilson chains via tridiagonalization, with local dissipators filling one chain and emptying the other. The hopping amplitudes along the chains away from the impurity initially exhibit exponential decay due to the logarithmic discretization at large energies (red), until they cross over into more uniform hopping amplitudes of order $\delta$ in the linear discretization regime (blue). (e) The sites in the logarithmic sector, together with the impurity, are numerically integrated out in standard NRG spirit. This provides an effective subspace for the low-energy description in terms of an effective renormalized impurity (RI) with multiple dissipators. (e') This is achieved by collecting all the logarithmic sector sites into a single Wilson chain (via a retridiagonalization) for the sake of numerical stability of the subsequent iterative diagonalization by NRG. The fixed number of states coming out of the last NRG iteration constitute the RI low-energy subspace.

i.e., either as a solution of a linear equation (left-hand side), or as the state to which an arbitrary initial state decays to in the long-time limit (right-hand side). The uniqueness of the solution stems from the driving of the leads, which is explicitly designed to drive each discretized lead level to its unique thermal equilibrium state in the absence of the impurity. The uniqueness of the steady state should not be affected by the introduction of the impurity, which contains only a small number of degrees of freedom. This is demonstrated for the noninteracting case in Appendices C and D. Adding interactions is not expected to impair this.

The first stage of the RL-NESS method is obtaining a Lindblad equation for a discrete system from the original continuous system, as shown in Fig. 2(b). Formally, this can be done by dividing the full band $[-D,+D]$ of each lead into consecutive distinct intervals $I_{n}$. By the bilinear structure of the coupling in Eq. (5), the impurity couples to a particular mode in each interval, which itself is then coupled to the remainder of the modes in that interval. The latter can be integrated out, leaving a single representative level for each interval. Explicitly performing this integration (under the Born and Markov approximations) yields the structure of the Lindblad baths and their couplings to the system. However, we will allow ourselves some freedom in choosing the exact values of the couplings to the Lindblad baths so as to simplify the subsequent simulation of the driven system, while enforcing that the correct steady state is obtained.

The choice of the intervals $I_{n}$ and corresponding energy levels relates to coarse graining that depends on a discretization scheme. A common discretization scheme used for treating quantum impurity models is the logarithmic discretization scheme, introduced by Wilson as part of NRG $[3,4]$. This scheme produces discrete semi-infinite leads with level spacing shrinking exponentially as the lead Fermi energy is approached. It is designed to generate energy scale separation, and subsequently justifies integrating out of high-energy modes via iterative exact diagonalization as part of a logarithmic RG flow. This enables us to accurately and reliably resolve exponentially small energy scales which frequently arise in impurity models due to Kondo-type physics. However, for an open system, e.g., due to coupling to a thermal reservoir or the presence of finite voltage bias, energy scale separation ceases to exist below the corresponding energy scale, and the logarithmic RG flow will be cut off. In the nonequilibrium case this gives rise to a dynamical low-energy window described by a reduced bandwidth $D^{*}$, which is of order of the bias voltage or temperature (see below). For a least-biased numerical approach then, the discretization scheme within this 
regime should be uniform. Therefore, RL-NESS employs a mixed discretization scheme [30,37]. This consists of a logarithmically discretized region extending from the band edge down to just above the lead bias voltage or temperature, that smoothly crosses over into a linearly discretized region (with uniform level spacing) in the bias window $\left[-D^{*},+D^{*}\right]$. Such a scheme allows one to make use of NRG to integrate out high-energy modes (relative to $V$ or $T$ ), in order to obtain an effective low-energy nonequilibrium system, to be simulated in a controlled manner by a DMRG-like approach. This scheme has also been discussed for the setup of two leads with a voltage or temperature bias in a previous work [30], but without the Lindblad driving (previously suggested in Ref. [32]). It is therefore briefly outlined here for completeness.

We define $D^{*}$, the characteristic energy scale of the leads, as the energy at which the Fermi-Dirac distribution of the lead drops below some preselected threshold. For zero temperature this implies $D^{*}=\frac{V}{2}$, while for finite temperature the specific value of $D^{*}$ depends on the chosen threshold. The intervals $I_{n}$, as shown in Fig. 2( $\left.\mathrm{b}^{\prime}\right)$, are chosen such that in the range $\left[-D^{*},+D^{*}\right]$ they are of equal size $\delta$, referred to as the linear discretization parameter, while away from this range they scale exponentially as $\sim \Lambda^{n}$, where $\Lambda>1$ is referred to as the logarithmic discretization parameter. In the intermediate region the interval widths cross over smoothly between being constant and growing exponentially. In each interval a representative energy level $\varepsilon_{n}$ is selected with corresponding coupling $v_{\alpha n \lambda}$ to the impurity $\lambda$ th level. For details regarding the choice the intervals and corresponding energies and couplings, see Appendix A. The same intervals are chosen for both leads such that by construction $\varepsilon_{n}$ are lead independent, while the coupling constants $v_{\alpha n \lambda}$ can differ between the leads. The resulting leads and coupling Hamiltonians are

$$
\begin{gathered}
H_{\text {leads }}^{(\text {disc })}=\sum_{\alpha, n} \varepsilon_{n} c_{\alpha n}^{\dagger} c_{\alpha n}, \\
H_{\text {coupling }}^{\text {(disc) }}=\sum_{\alpha, \lambda} \underbrace{\sum_{n} v_{\alpha n \lambda}\left(c_{\alpha n}^{\dagger}\right.}_{\equiv t_{\alpha 0 \lambda} c_{\alpha 0}^{\dagger}} d_{\lambda}+\text { H.c. }) .
\end{gathered}
$$

Following through with this procedure, the dissipators can formally be derived. If such a path is pursued, the continuum of modes of a specific interval will serve as the environment only of its representative level, thus resulting in a local dissipator for each level:

$$
\begin{aligned}
\mathcal{D}_{\alpha n} \rho= & \gamma_{n}\left[1-f_{\alpha}\left(\varepsilon_{n}\right)\right]\left(2 c_{\alpha n} \rho c_{\alpha n}^{\dagger}-\left\{c_{\alpha n}^{\dagger} c_{\alpha n}, \rho\right\}\right) \\
& +\gamma_{n} f_{\alpha}\left(\varepsilon_{n}\right) \quad\left(2 c_{\alpha n}^{\dagger} \rho c_{\alpha n}-\left\{c_{\alpha n} c_{\alpha n}^{\dagger}, \rho\right\}\right),
\end{aligned}
$$

where $f_{\alpha}(\varepsilon) \equiv f_{\mathrm{FD}}\left(\varepsilon ; \mu_{\alpha}, T_{\alpha}\right)$ is the Fermi-Dirac distribution for lead $\alpha$ (depending on the lead specific chemical potential and temperature), and $\left\{\gamma_{n}\right\}$ are referred to as Lindblad-driving rates. This structure implies that when the leads are decoupled from the impurity, i.e., $v_{\alpha n \lambda}=0$, they are driven to their equilibrium occupation, as expected. The total Lindblad equation is then given by

$$
\mathcal{L} \rho=-i\left[H_{\text {total }}^{(\mathrm{disc})}, \rho\right]+\sum_{\alpha n} \mathcal{D}_{\alpha n} \rho,
$$

where $H_{\text {total }}^{(\text {disc }} \equiv H_{\text {dot }}+H_{\text {leads }}^{(\text {disc }}+H_{\text {coupling }}^{(\text {disc })}$ is the total Hamiltonian, now with discrete leads, and hence effectively of a finite system that becomes an open system by means of the Lindblad driving. As shown in Refs. [32,38,39], a wide range of driving rates reproduce the same continuum limit observables. Therefore, one is free to choose them, in this range, so as to best suit the numerics. With this in mind, and for reasons to be explained in Sec. IIC, the rates will all be chosen to be energy independent, i.e., $\gamma_{n}=\gamma$, and of order of the linear level spacing $\delta$. Let us note that driving of energy modes (exponentially) larger than $D^{*}$ will have negligible effect on the results since, importantly, these modes start and practically remain in equilibrium throughout the dynamics. Thus, the corresponding rates can further be tuned, or even completely turned off, in order to enhance numerical stability, as we further discuss in Sec. III and Appendix F. At this point, the Lindblad equation to be solved is fully defined. As a consistency check, note that properly taking the limits of this equation converges back to the continuum limit: In the limit $\Lambda \rightarrow 1$ the discretization scheme collapses to a linear (equal spacing) discretization, which in turn converges to the continuous system in the $\gamma=\delta \rightarrow 0$ limit [32].

\section{Local form}

The Lindblad-driven discretized levels (LDDL) scheme [32] is a set of exact manipulations, applied to the Lindblad equation (11) with the goal of bringing it to a form more favorable for treatment in the framework of tensor networks. The system Hamiltonian obtained after discretization is formulated in the so-called star geometry, involving diagonal leads, as in Eq. (8), with all levels directly coupled to the impurity, as in Eq. (9). This geometry is nonlocal (in the sense that all lead levels couple to the impurity), and therefore less convenient in the framework of tensor networks. The dissipators, on the other hand, are already local in this geometry, with each lead level coupled to its own Lindblad bath, which is a property we would like to retain. A standard procedure, employed for example in NRG, is to perform an exact mapping in terms of a basis transformation from the star geometry to a chain geometry [4]. The bilinear structure of the coupling term in Eq. (9) directly defines the only bath level $c_{\alpha 0}^{\dagger}$ that the impurity couples to. This level constitutes the first site of a nearest-neighbor tight-binding chain, which can be obtained by tridiagonalizing the single-particle basis of the remainder of the lead levels, e.g., by construction of a full Krylov space:

$H_{\text {leads }}^{\text {(disc) }}=\sum_{\alpha, k} t_{\alpha k}\left(c_{\alpha k}^{\dagger} c_{\alpha k+1}+c_{\alpha k+1}^{\dagger} c_{\alpha k}\right)+\sum_{\alpha, k} \varepsilon_{\alpha k} c_{\alpha k}^{\dagger} c_{\alpha k}$.

Such a basis transformation, however, will result in nonlocal dissipators due to the $n$-dependent prefactors in Eq. (10), which include the Fermi factors. The LDDL scheme circumvents this problem and yields a Lindblad equation which is local in both the dissipators and the Hamiltonian in the chain geometry. For completeness it will be described here briefly. The idea behind this scheme is to shift the Fermi-Dirac information from the dissipators in Eq. (10) to the lead-impurity couplings in an effective Hamiltonian, still in the star geometry. With an appropriate choice of Lindblad-driving rates, the 
system can then be tridiagonalized into the chain geometry, without losing the locality of the dissipators.

Consider a single discrete lead level with creation operator $c_{\alpha n}^{\dagger}$, referred to as a physical level, in lead $\alpha$ at energy $\varepsilon_{n}$ and coupling constants $v_{\alpha n \lambda}$ to the impurity levels. We temporarily drop the subscripts $\alpha n$ for readability in what follows. Its dissipator is given according to Eq. (10), meaning it is constantly depopulated and repopulated at a constant Lindblad-driving rate $\gamma$, weighted by $1-f(\varepsilon)$ and $f(\varepsilon)$, respectively. In the LDDL scheme this single physical level is mapped onto two artificial lead levels with creation operators $c_{h}^{\dagger}$ and $c_{p}^{\dagger}$, referred to as hole and particle levels, thus effectively doubling the number of levels. The former is constantly depopulated at rate $\gamma$ and the latter constantly repopulated at rate $\gamma$ :

$$
\begin{aligned}
& \mathcal{D}_{h} \rho=\gamma\left(2 c_{h} \rho c_{h}^{\dagger}-\left\{c_{h}^{\dagger} c_{h}, \rho\right\}\right), \\
& \mathcal{D}_{p} \rho=\gamma\left(2 c_{p}^{\dagger} \rho c_{p}-\left\{c_{p} c_{p}^{\dagger}, \rho\right\}\right) .
\end{aligned}
$$

These two levels both have the same onsite energy $\varepsilon$, yet are now coupled to the impurity with amplitudes that depend on temperature and chemical potentials:

$$
v_{\lambda, h}=\sqrt{1-f(\varepsilon)} v_{\lambda}, \quad v_{\lambda, p}=\sqrt{f(\varepsilon)} v_{\lambda} .
$$

Formally this mapping is obtained by introducing an auxiliary level at energy $\varepsilon$ which is decoupled both from the impurity and the Lindblad baths, and performing a unitary rotation between the physical and auxiliary levels, thus shifting the Fermi-Dirac information from the dissipators to the leadimpurity couplings, as shown in Fig. 2( $\left.\mathrm{c}^{\prime}\right)$. For more details, as well as a discussion of the resemblance of this procedure to purification of the level, or the thermofield approach, see Ref. [30].

The described procedure is repeated for each lead level. This replaces each physical lead with a corresponding hole lead and particle lead, as in Fig. 2(c), thus doubling the total number of lead levels. By selecting Lindblad-driving rates $\gamma$ to be energy independent, one obtains dissipators for each of the hole or particle leads which do not depend on the energy index $n$ (i.e., are proportional to to the identity matrix with respect to this index). Each such lead can therefore be tridiagonalized separately into a nearest-neighbor chain while the dissipators remain unaltered, resulting in a Lindblad equation which is local both in the dissipators and the Hamiltonian, as desired [see Fig. 2(d)].

Two remarks are in order regarding the doubling of lead levels, before the tridiagonalization is performed. The first is that for physical levels lying far from the chemical potential in units of temperature, where $f(\varepsilon)$ is $0(1)$, the particle (hole) level decouples from the impurity, and can thus be disregarded in subsequent calculations. For zero temperature this holds for all physical levels, and so the described mapping is reduced to relabeling physical levels above (below) the lead chemical potential as holes (particles), with no doubling actually occurring.

The second remark relates to exploiting a left-right symmetry in the lead spectrum. In equilibrium calculations, when both leads have the same energy levels and for each lead level the left and right coupling constants to all impurity levels are proportional, only a specific linear combination of left and right levels couples to the impurity, precisely as defined by the coupling Hamiltonian. The complementary orthogonal combination of left and right levels decouples from the impurity and hence becomes irrelevant for the impurity dynamics. This simplifies the model from a two-lead model to an effective single-lead model. In the nonequilibrium case, the different potentials applied to the left and right leads break this symmetry, and prevent its exploitation. However, once the physical leads are separated into hole and particle leads, the symmetry is reinstated (for holes and particles separately), and can therefore be exploited. Thus, for models in which this symmetry exists, the final number of artificial lead levels is actually smaller than the original number of physical levels.

\section{Renormalized impurity: NRG}

The LDDL scheme is indifferent to the specific discretization scheme employed, as long as the Lindblad-driving rates are kept energy independent. Observe now the implications of the linear-logarithmic scheme on the resulting Lindblad equation. The obtained onsite energies $\left\{\varepsilon_{\alpha k}\right\}$ and nearestneighbor hopping amplitudes $\left\{t_{\alpha k}\right\}$ in the vicinity of the impurity are of the largest magnitude and decay exponentially as the distance from the impurity grows, all the way down to $D^{*}$. The corresponding sites will therefore be referred to as the logarithmic sector. Below $D^{*}$, the onsite energies and hopping amplitudes remain of order of the linear level spacing $\delta$ and $D^{*}$, respectively, and will be referred to as the linear sector. Due to the smooth transition in the discretization, the exact boundaries between these two sectors are fuzzy, and in practice are chosen with some fine tuning in order to enhance convergence.

In the chain geometry, the logarithmic sector, including the impurity, can be considered as a mesoscopic system, coupled to the linear sector leads. By construction, the vast majority of the (many-body) energy levels of this mesoscopic system are at energies larger than $D^{*}$, and so are expected to be indifferent to the voltage bias applied, thus largely remaining in the equilibrium state. They are therefore expected to contribute to the nonequilibrium dynamics only through renormalization effects on the low-energy modes in the linear sector, which in turn actively participate in the dynamics. As argued in Ref. [30], it is therefore sufficient to approximate the mesoscopic system by a renormalized impurity (RI) residing in an effective significantly reduced low-energy basis. This is a controlled approximation, as one can monitor the weight on all states in the RI while time evolving toward $\rho_{\mathrm{NESS}}$. Note that the chemical potential of this RI is set midway between the chemical potentials of the leads, so that the effective lowenergy subspace consists of states with RI particle number which is close to its average occupation in the NESS.

The RI is obtained by the following procedure, as described in Fig. 2( $\left.\mathrm{e}^{\prime}\right)$ : an additional subsequent tridiagonalization is applied to merge the two (particle and hole) chains in the logarithmic sector. This brings them into a single-lead Wilsonchain structure, which is important for NRG since it keeps correlations at a given energy scale local. An NRG sweep is then applied to the chain: starting from the impurity, at each 
step a site is added to the chain, the Hamiltonian is diagonalized, and high-energy modes are discarded. At the end of the sweep through the logarithmic sector, the $R$ lowest-lying many-body states are taken as the effective basis of the RI. All operators acting on the impurity, or on sites in the logarithmic sector, are then projected to this effective reduced basis.

The leads in the linear sector, together with the RI, now form the dynamical system under consideration, as shown in Fig. 2(e). The Lindblad equation for this system still consists of a nearest-neighbor Hamiltonian, however, with a more complicated local term acting on the RI site. The dissipators are also local in this setup, and again the local terms acting on the RI are more complicated, corresponding to the multiple dissipators acting on the logarithmic sector. Note that although the dissipators on different sites of the chain originally commute, the logarithmic sector dissipators, after being projected to the RI basis, no longer do. Another concern regarding the logarithmic sector dissipators is that because they were not taken into account during the RG flow, they might drive the RI out of the effective low-energy basis. In practice, this issue can be handled, as discussed below in Sec. III and Appendix F.

\section{E. MPDO solution: tDMRG}

The obtained Lindblad equation is solved for the steady state by real-time evolution, implemented in the tensornetwork formalism. The system (mixed) state is represented as a matrix-product density operator (MPDO) [40,41]. Analogously to the matrix-product state (MPS) representation of wave functions, which has a single (physical) index for each chain site, the MPDO has two (physical) indices for each chain site, as shown in Fig. 3(a). For the chain at hand, each of these physical indices is of dimension $d$ corresponding to a single fermionic Hilbert space, except at the RI, where it is of dimension $R$ corresponding to the effective low-energy subspace. It is common practice to combine the two physical indices of each MPDO site into a single effective index of dimension $d^{2}$ and simply treat it as an MPS. However, in the case of a large physical index dimension, e.g., for the RI, keeping the indices separate enables more efficient contractions and reduces the computation cost.

Local symmetries in the continuous system imply charge conservation. By construction, the derivation of the Lindblad equation will respect these symmetries, so that the Lindblad dynamics conserves the corresponding charges in the full system, i.e., the discrete system together with the baths. However, it does not necessarily conserve these charges in the discrete finite system alone. Still, the fact that the symmetries are respected results in related conserved quantities in the discrete system $[42,43]$, which can be exploited in order to decompose the MPDO into symmetry sectors, further reducing the computational cost. For concreteness we demonstrate this for a $\mathrm{U}(1)$ charge (particle number) conserved in the original continuous system, and the generalization to other symmetries is straightforward. Define the superoperators $\mathcal{N}_{ \pm}=$ $N \otimes \mathbb{I} \pm \mathbb{I} \otimes N$, where $N$ is the particle-number operator, $\mathbb{I}$ is the identity, and $A \otimes B$ is understood to operate on to the density operator as $A \rho B$. The Liouvillian superoperator $\mathcal{L}$

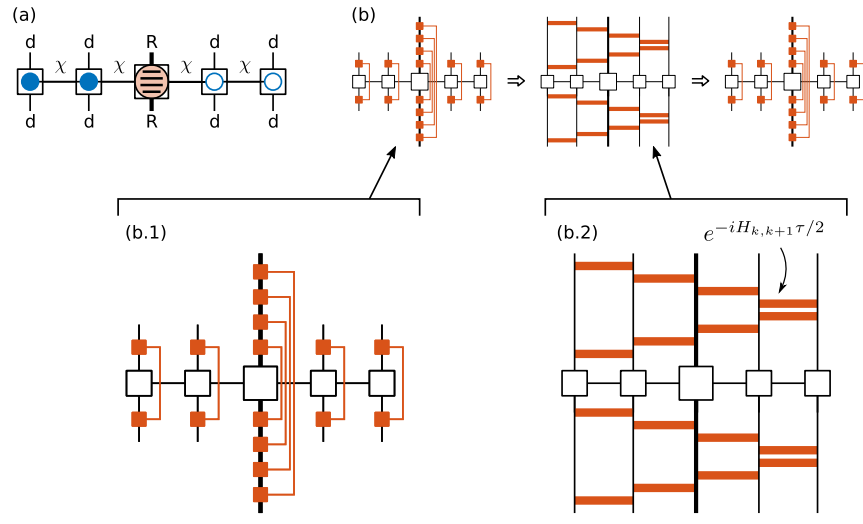

FIG. 3. (a) MPDO description of the system density operator: each site is described by a rank-4 tensor (i.e., 4 legs) with two physical indices, of local dimension $d$ for chain sites or $R$ for the renormalized impurity (RI), and two virtual indices, of bond dimension $\chi$, connecting it to its neighboring tensors. The initial MPDO is chosen as a product state, where all particle sites are full, all hole sites are empty, and the RI is in its ground state. (b) A single second-order Trotterized time step consists of a dissipative half-time-step sweep (b.1), a Hamiltonian full time-step sweep (b.2: sweep forward and backward at $\tau / 2$ ), and then another dissipative half-time-step sweep in the opposite direction [cf. Eq. (16)]. (b.1) Dissipative evolution half-time-step sweep: each red square corresponds to the set of Kraus gates applied to a specific site, and the sum on all gates at that site is implied by the red contraction line (see text). At the RI, multiple gates are sequentially applied, corresponding to the multiple driven sites incorporated into it. (b.2) Hamiltonian evolution time step: each red bar corresponds to a half-time-step Trotter gate $e^{ \pm i H_{k, k+1} \tau / 2}$ [cf. Eq. (17)] applied to sites $k, k+1$. The gates are applied from left to right and then back from right to left for a full time step.

commutes with $\mathcal{N}_{-}$but not with $\mathcal{N}_{+}$. The dynamics therefore does not conserve particle number in the discrete system $N \otimes \mathbb{I}=\frac{\mathcal{N}_{+}+\mathcal{N}_{-}}{2}$. However, the conservation of $\mathcal{N}_{-}$suffices in order to decompose the MPDO into particle-number symmetry sectors. It also implies that the parity of $\mathcal{N}_{+}$and thus of $N \otimes \mathbb{I}$ is conserved, which suffices in order to account locally for fermionic signs.

Following Ref. [30], the system is set in an initial state $\left|\psi_{0}\right\rangle$ which is a product state between the ground state of the decoupled RI, and the steady state of the decoupled linear sector leads. The latter is defined as the pure product state where all lead particle (hole) sites are full (empty). This initial state can be written either as an MPS, or as an MPDO for $\rho_{0} \equiv\left|\psi_{0}\right\rangle\left\langle\psi_{0}\right|$, both with bond dimension $\chi=1$. This starting point is assumed to be sufficiently close to the desired final steady-state solution, so that when the coupling to the RI is turned on, the full system will quickly converge to its steady state (as our results confirm). One could also initialize the RI in its decoupled steady state, but in practice this does not improve convergence. Note that the initial setup, together with its transient dynamics, are regarded only as a means to obtain the desired steady state, so that the specific choice of initial state can be fully based on numerical considerations. 
The coupling between the RI and the leads is then turned on, and the system is evolved in time by a variant of tDMRG, formulated to accommodate for Lindblad dynamics. Note that in this work the RI-lead coupling is turned on in an immediate quench, and slow ramping up of the coupling, as employed in Ref. [30], was not necessary. In the spirit of tDMRG, this time evolution is based on a second-order Trotter-Suzuki decomposition with a sufficiently small time step $\tau$. Then, the propagator can be written as a product of short-time propagators $e^{\mathcal{L} t}=\prod_{i=1}^{N_{t}} e^{\mathcal{L} \tau}$, with $N_{t}$ steps required in order to arrive at a time $t=\tau N_{t}$. Each short-time propagator is Trotter decomposed into local and nearest-neighbor gates based on the short rangedness of the Liouvillian introduced above. The total Hamiltonian can be written as a sum of local two-site operators $H=\sum_{k=1}^{N-1} H_{k, k+1}$ where nonadjacent terms commute. Defining the Hamiltonian two-site superoperators as $\mathcal{H}_{k, k+1} \rho \equiv-i\left[H_{k, k+1}, \rho\right]$, the Liouvillian $\mathcal{L}$ can then be written as the sum of these two-site Hamiltonian terms and single-site dissipative (hole/particle) terms defined in Eq. (13):

$$
\mathcal{L}=\sum_{k=1}^{N-1} \mathcal{H}_{k, k+1}+\sum_{k=1}^{N} \mathcal{D}_{k}
$$

For an exact representation of the superoperators, the Hamiltonian terms commute with all nonadjacent Hamiltonian and dissipative terms, and the dissipative terms all commute with each other. However, inside the RI the fermionic anticommutation relations of the original fermionic operators are compromised by the NRG truncation, which results in noncommuting terms in its vicinity. The second-order Trotter decomposition adopted here and depicted in Fig. 3(b) is similar to the one discussed in Ref. [44]:

$$
e^{\mathcal{L} \tau} \approx \underbrace{\prod_{k=1}^{N} e^{\frac{\tau}{2} \mathcal{D}_{k}}}_{\text {Fig. 3(b.1) }} \underbrace{\prod_{k=N}^{2} e^{\frac{\tau}{2} \mathcal{H}_{k-1, k}} \prod_{k=1}^{N-1} e^{\frac{\tau}{2} \mathcal{H}_{k, k+1}}}_{\text {Fig. 3(b.2) }} \underbrace{\prod_{k=N}^{1} e^{\frac{\tau}{2} \mathcal{D}_{k}}}_{\text {Fig. 3(b.1) }} .
$$

The two-site Hamiltonian gates are given by

$$
e^{\frac{\tau}{2} \mathcal{H}_{k, k+1}} \rho \equiv e^{-i \frac{\tau}{2} H_{k, k+1}} \rho e^{i \frac{\tau}{2} H_{k, k+1}},
$$

and the dissipative single-site gates translate into Kraus operators $[45,46]$. In the spinless case they are respectively given for particles or holes by

$$
\begin{array}{ll}
e^{\frac{\tau}{2} \mathcal{D}_{\eta}} \rho=K_{1 \eta} \rho K_{1 \eta}^{\dagger}+K_{2 \eta} \rho K_{2 \eta}^{\dagger}, & \eta \in\{h, p\}, \\
K_{1 h}=e^{-\frac{\gamma \tau}{2} c^{\dagger} c}=c c^{\dagger}+e^{-\frac{\gamma \tau}{2}} c^{\dagger} c, & K_{2 h}=\sqrt{1-e^{-\gamma \tau}} c, \\
K_{1 p}=e^{-\frac{\gamma \tau}{2} c c^{\dagger}}=c^{\dagger} c+e^{-\frac{\gamma \tau}{2}} c c^{\dagger}, & K_{2 p}=\sqrt{1-e^{-\gamma \tau}} c^{\dagger} .
\end{array}
$$

For spinful fermions there will be four Kraus operators for each site, replacing $\eta \rightarrow(\eta, \sigma)$, with $\sigma \in\{\uparrow, \downarrow\}$. For terms which are bilinear in the fermionic operators, such as the Hamiltonian or $K_{1 \eta}$, fermionic signs arising from the anticommutation relations can be accounted for locally. The operators $K_{2 \eta}$ in the dissipative terms, however, act simultaneously on both sides of the density matrix. Hence, they give rise to a global Jordan-Wigner string. In the present MPDO setup, it can be efficiently "pulled" in locally [47]. This requires that charge parity is fully tracked on all tensors, which is the case here when decomposing the MPDO into U(1) charge symmetry sectors, in the sense discussed above. In the local configuration, as shown in Fig. 3(b.1), the crossing of the red line with the bond index implies that the charge parity operator $Z \equiv(-1)^{q}$, with charge $q$, must be simultaneously applied to the bond state space when acting with $K_{2 \eta}$.

A quick overall complexity analysis of the method can be performed assuming a fixed bond dimension $\chi$ on all MPDO sites. Since the treatment of the RI is clearly the most expensive step, the following considers operations involving the RI. The analysis is completely analogous for all other sites where one simply replaces $R$ with the regular local dimension $d$ of a physical site. The cost of the Trotter gate contraction is $O\left(d^{2} R^{2} \chi^{3}+d^{3} R^{3} \chi^{2}\right)$, where the two terms correspond to merging the RI tensor with its neighboring tensor and to applying the nearest-neighbor Trotter gate, respectively. The SVD back into local tensors then costs $O\left(d^{4} R^{2} \chi^{3}\right)$. Finally, the cost of the Kraus gate contractions is $O\left(k R^{3} \chi^{2}\right)$, where $k$ is the number of Kraus gates acting on the RI, which is proportional to the number of sites in the logarithmic sector. Ignoring the cost of all other sites in the linear sector, the total cost of the method can be approximated as $O\left(N_{t}\left(d^{3} R+k R+d^{4} \chi\right) R^{2} \chi^{2}\right)$, where $N_{t}$ is the number of sweeps.

The most important property of the MPDO ansatz is that it can efficiently represent the steady state, using a relatively small number of parameters. Another important constraint on the represented state is that it must be a physical state, i.e., a positive-semidefinite Hermitian operator with finite trace. While the MPDO ansatz does not enforce these constraints, the Lindblad evolution (also after Trotterization) is a completely positive trace-preserving (CPT) map [46], and thus guarantees that starting from a physical state will always result in a physical state. The only loss of positivity (and trace) can come from truncation of singular values during the tDMRG sweep. This relates to a drawback of the MPDO ansatz: the singular values obtained after a Schmidt decomposition no longer correspond to the singular values of the reduced density matrix (as for an MPS). However, if the singular values drop quickly enough, as is the case for the models analyzed here, only small singular values are truncated, resulting in negligible loss of positivity.

\section{F. Observables}

At any point throughout the evolution, single-time correlations can be extracted from the MPDO, with the long-time limit representing the steady-state value. As correlations in the chain geometry are easily obtained, in practice, it is convenient to map all observables of interest to this geometry, as shown in Ref. [30]. In this work we focus on the particle current flowing from one lead to the other. The time derivative of the impurity occupation can be separated into contributions $I_{\alpha}$ corresponding to the current flowing from lead $\alpha$ into the impurity:

$$
-e \frac{d}{d t}\left\langle n_{d}\right\rangle=-\frac{e i}{\hbar}\left\langle\left[H, n_{d}\right]\right\rangle=\sum_{\alpha} I_{\alpha} .
$$



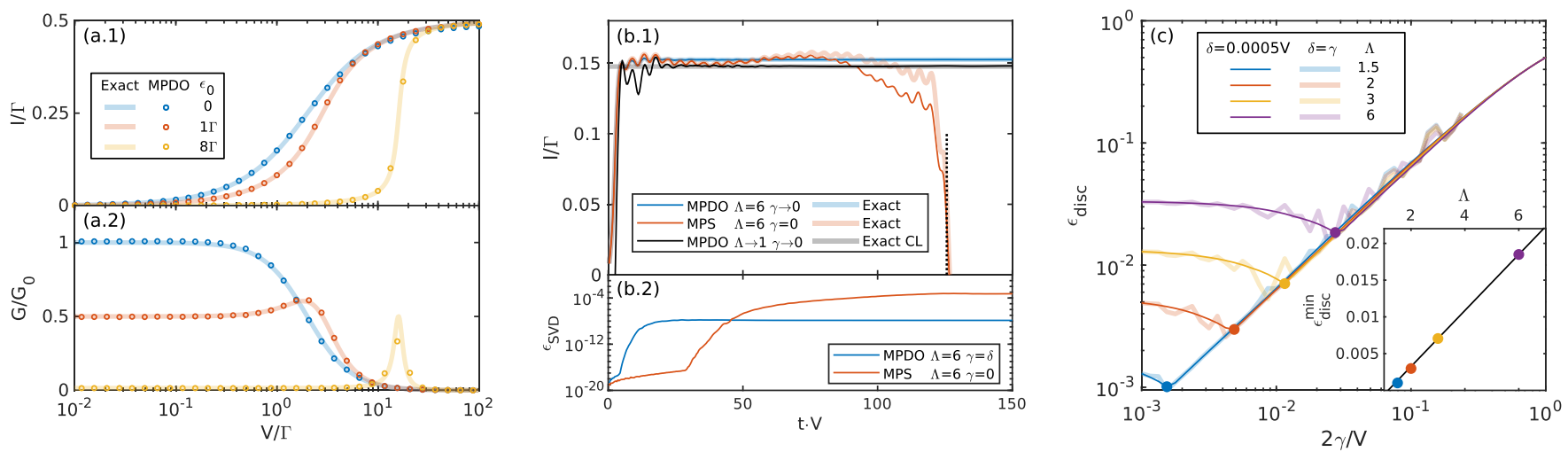

FIG. 4. (a.1) NESS current and (a.2) differential conductance of the RLM (in units of the conductance quantum $G_{0}=e^{2} / h$ ) as a function of bias voltage $V$ and at different gate voltages $\varepsilon_{0}$, as calculated exactly (solid lines) and by RL-NESS (circles). Simulation results are obtained for $\delta=0.1 D^{*}, D^{*}=\frac{V}{2}$ and after linear extrapolation in $\gamma / \delta=2,1 \rightarrow 0$ and $\Lambda=8,6 \rightarrow 1$, with bond dimension $\chi=256$ and $R=32$ states kept in the RI. (b.1) Comparison of the RL-NESS MPDO evolution $(\chi=256$ ), after extrapolation to $\gamma \rightarrow 0$ (blue), and the NRG-tDMRG MPS evolution $(\chi=1024)$ for $\gamma=0$ (red), both with the same discretization, at bias voltage $V=\Gamma$ and $\varepsilon_{0}=0$. Exact results for both cases are also plotted (shaded). The MPDO result is then further linearly extrapolated from $\Lambda=8,6$ to $\Lambda=1$ (solid black) and compared to the exact continuous leads (CL) result (shaded gray). (b.2) Truncation error (sum of singular values squared accumulated over several time steps) plotted for the RL-NESS MPDO with $\gamma=\delta$ (blue) and the NRG-tDMRG MPS (red) evolution. (c) Discretization error $\epsilon_{\mathrm{disc}}$ for the RLM at $\varepsilon_{0}=0$ as a function of $\gamma$ for different values of $\Lambda$, with $\delta=0.0005 \mathrm{~V}$ (solid) and $\delta=\gamma$ (shaded). The inset displays the $\Lambda$ dependence of the lower bound on the discretization error $\epsilon_{\text {disc }}$ (corresponding to the circles in the main figure).

Thus, $I_{\alpha}$ is given in the continuum limit (taking $e=1, \hbar=1$ ), and approximated after discretization by

$$
I_{\alpha}=2 \sum_{\lambda} v_{\alpha \lambda} \int_{-D}^{D} \frac{d \varepsilon}{\sqrt{2 D}} \operatorname{Im}\left\langle c_{\alpha \varepsilon}^{\dagger} d_{\lambda}\right\rangle \approx 2 \sum_{n, \lambda} v_{\alpha n \lambda} \operatorname{Im}\left\langle c_{\alpha n}^{\dagger} d_{\lambda}\right\rangle .
$$

In the steady state $\frac{d}{d t}\left\langle n_{d}\right\rangle=0$, the current flowing from the left lead into the impurity is equal to the current flowing from the impurity into the right lead $I \equiv I_{L}=-I_{R}$. From a numerical perspective, the average combination $I=\left(I_{L}-I_{R}\right) / 2$ converges more rapidly, and is less prone to noise. Running simulations for different voltages, a full $I-V$ curve can be obtained, and numerically differentiated in order to produce the differential conductance $G(V)=\Delta I / \Delta V$. Note that the numerical derivative is very sensitive to noise, so that the $I-V$ curve must be obtained with high accuracy.

\section{ERROR ANALYSIS AND THE RLM}

In order to estimate the accuracy of the presented method, we apply it to the exactly solvable noninteracting resonant level model (RLM). This model, with dot Hamiltonian

$$
H_{\mathrm{dot}}^{\mathrm{RLM}}=\varepsilon_{0} n_{0},
$$

describes a single spinless impurity level with energy $\varepsilon_{0}$ (e.g., controlled by a gate voltage), coupled to two spinless leads described by Eq. (4) via the coupling Hamiltonian (5). An end-to-end comparison of the steady-state current and the differential conductance, between the exact result of the RLM in the continuum limit and the RL-NESS result, is shown in Fig. 4(a). It displays a good agreement over a wide range of bias voltages and impurity level energies $\varepsilon_{0}$, with parameter values given in the caption.
The RL-NESS real-time evolution of the current, for a typical case of $V=\Gamma, \varepsilon_{0}=0$ with finite $\Lambda=6$ and linearly extrapolated to $\gamma \rightarrow 0$, is plotted in Fig. 4(b) (blue). It demonstrates several key aspects of the method. After an initial rapid rise in the current over a period of order $V^{-1}$, the oscillations (discretization artifacts related to the logarithmic sector) decay exponentially at a rate which is proportional to $\gamma$, finally stabilizing on a steady-state value. For further discussion regarding the evolution timescales, see Appendix D. The convergence to the steady state can also be observed in the lower panel, where the truncation error saturates. Throughout the entire evolution, our method displays excellent agreement with the corresponding exact result (shaded blue) of the same driving protocol. After linearly extrapolating also to the $\Lambda \rightarrow 1$ limit, the RL-NESS current (black), once the NESS is reached, displays excellent agreement with the continuum limit exact result (shaded gray).

If the dissipation is initially set to $\gamma=0$, while keeping a finite level spacing, RL-NESS reduces to the NRG-tDMRG scheme [30], in which the state of the system is represented by an MPS (instead of an MPDO), and the real-time evolution is unitary. In what follows, this will simply be referred to as MPS evolution. As in the case of finite dissipation, the $\gamma=0$ evolution of the current can be calculated (for the same $\Lambda=6$ ) either explicitly as an MPS evolution (solid red) or exactly in the single-particle basis (shaded red). These results agree with the RL-NESS current in the early transient oscillatory regime, but later residual oscillations persist. Thus, only a quasi-steady state is obtained, whose mean is nevertheless consistent with the $\gamma \rightarrow 0$ limit. Eventually, the current drifts away, due to truncation errors that, without dissipation, do not saturate. Later on, even for the exact solution, this quasi-steady state will be lost due to reflection off the edges of this closed system at a time $t=L / v_{F}=\frac{2 \pi}{\delta}$, dictated by the finite linear level spacing. In stark contrast, in the case 
of RL-NESS, for strong enough damping $\gamma \lesssim \delta$, the discrete levels become sufficiently blurred out, such that the dynamics truly represents an open system, where reflection off the edges and the accompanying drop in the current no longer occur.

As part of the analysis, steady-state observables of the RLM are calculated exactly by means of Keldysh formalism, both for the continuous system and in an arbitrary discretization (Appendix B). The exact time evolution of single-time observables (in a given discretization) is also calculated by solving a differential continuous Lyapunov equation for the single-particle correlation matrix (Appendix C).

The remainder of this section is dedicated to an analysis of the two major error contributions in the method: the lead discretization error (how well do the discrete system observables represent the continuous system), and the simulation error (how accurately does the tensor-network method solve for the discrete system steady state). Generally, there is a tradeoff between the two contributions, as a finer discretization better reproduces the continuum limit, but is also harder to solve for numerically.

The lead discretization error depends both on the fineness of the discretization grid, controlled by the logarithmic $\Lambda$ and linear $\delta$ discretization parameters, and on the broadening of the discrete levels, controlled by the Lindblad driving rates $\gamma$. We introduce the relative measure for the discretization error $\epsilon_{\text {disc }} \equiv \max _{V}\left|1-I_{\mathrm{DL}} / I_{\mathrm{CL}}\right|$, as the maximal relative distance over a range of bias voltages $V \in[0.01,100] \Gamma$, between the exact discrete leads current $I_{\mathrm{DL}}$ (for a specific choice of $\Lambda$ and ratios $\delta / V, \gamma / V)$ and the exact continuous leads current $I_{\mathrm{CL}}$. This measure can be explicitly evaluated for the RLM and is plotted in Fig. 4(c) as a function of $\gamma / V$ and for several values of $\Lambda$. The specific choice of $\delta$ has only a minor effect, as long as $\delta \leqslant \gamma$, which is required in order to negate finitesize effects. Fixing $\delta / V$ to a small value results in a smooth curve (solid), while taking $\delta=\gamma$ results in a slightly more noisy curve (shaded) with the same trend. Note that $\epsilon_{\text {disc }}$ is approximately linear in $\gamma / V$, down to a lower bound on the error, dictated by $\Lambda$. This lower bound in turn is linear in $\Lambda$ [see inset to Fig. 4(c)]. These two observations justify a linear extrapolation in these two parameters to the continuum limit $\Lambda \rightarrow 1, \delta=\gamma \rightarrow 0$ at each $V$.

The simulation error has multiple contributions, listed in ascending order of significance. First consider the Trotter error, arising from the discretization of the Liouvillian real-time evolution. In practice, exploiting second-order Trotter decomposition, it is numerically feasible to choose sufficiently small time steps such that this error is negligible compared to the other ones. A second source of simulation error is introduced by the NRG procedure, and controlled by the number of kept states in each NRG iteration. As in equilibrium, the number of required kept states can be reduced by taking a coarser logarithmic discretization, i.e., larger $\Lambda$. In practice, only the number of kept states $R$ in the last NRG iteration, dictating the size of the restricted low-energy subspace of the RI, poses a computational bottleneck. The numerical cost in setting up the RI by previous NRG iterations is entirely negligible. Therefore, earlier NRG iterations can be, and in practice are, less harshly truncated, but a larger $\Lambda$ is still required in any case in order to keep $R$ sufficiently small in the last iteration.
The third, and most significant, source of simulation error is the truncation of the MPDO to a fixed bond dimension $\chi$ after each time step, by discarding small singular values. Empirically, the singular values decay faster than polynomially with the singular value index, at a rate which decreases with decreasing $\gamma$ (see Appendix E). This implies that the required bond dimension (for a fixed truncation error) scales exponentially with $\gamma$. This exponential scaling can naturally be understood in the $\gamma \rightarrow 0$ limit, in which the entanglement entropy grows linearly in time, thus leading to an exponential blowup in the required bond dimension. Choosing a finite $\gamma$ sets a timescale $1 / \gamma$ at which the entanglement entropy stops growing. For any finite $\gamma$ the steady state can therefore be represented with a finite (possibly large) bond dimension, which in the small- $\gamma$ limit must grow exponentially with $1 / \gamma$ in order to match the expected exponential blowup. It is important to stress that even though there is an exponential bound on simulating small $\gamma$, this represents the thermodynamic limit, which can be approached by working with finite $\gamma$ and then linearly extrapolating to small $\gamma$.

Finally, let us discuss the issue of whether or not to apply the Lindblad terms coupled to the RI. Physically, since the RI represents the degrees of freedom far above the voltage and temperature bias scales, it is reasonable to expect that they are barely affected by the nonequilibrium conditions. Thus, whether or not the Lindblad terms acting on the RI are applied, we expect to obtain similar results. We demonstrate this for the RLM in Appendix F. Numerically, however, the effort involved in the two approaches (for the same accuracy) is different. The effect on the numerical results becomes more pronounced in the interacting case, considered in the next section. There, for a large logarithmic discretization parameter $\Lambda$, the RI spectrum contains nearly degenerate levels, which can be coupled even by weak Lindblad driving at the sites composing the RI. In practice this can drive, and hence affect, high-energy modes in the RI (which in principle should remain in equilibrium), resulting in artifacts which are enhanced in the differential conductance. Taking small values of $\Lambda \sim 2$ could resolve this problem. However, this necessitates increasing the number $R$ of states kept in the RI, and therefore is often impractical. Taking the manageable intermediate value $\Lambda=3$ for the interacting case (instead of extrapolating to $\Lambda \rightarrow 1$ ), at the cost of a larger $R=64$, suppresses these artifacts, but still does not completely eliminate them. For these reasons, in the interacting case it becomes preferable to entirely turn off the Lindblad terms coupled to the RI. This does not adversely affect the physics. On the contrary, it leads to a stable numerical solution without artifacts, with reasonable computational costs.

\section{INTERACTING SYSTEM}

We now wish to demonstrate the method on an interacting system, which has no known solution for the NESS current. For this we choose an interacting two-level model (I2LM), consisting of two interacting dot levels $\varepsilon_{1}, \varepsilon_{2}$ with onsite interaction energy $U$, coupled to noninteracting leads. The dot levels are taken with level spacing $\Delta \equiv \varepsilon_{2}-\varepsilon_{1}$, and can be shifted by changing $\varepsilon_{0}$ by a gate voltage (taken relative to particle-hole symmetry), such that the dot Hamiltonian is 

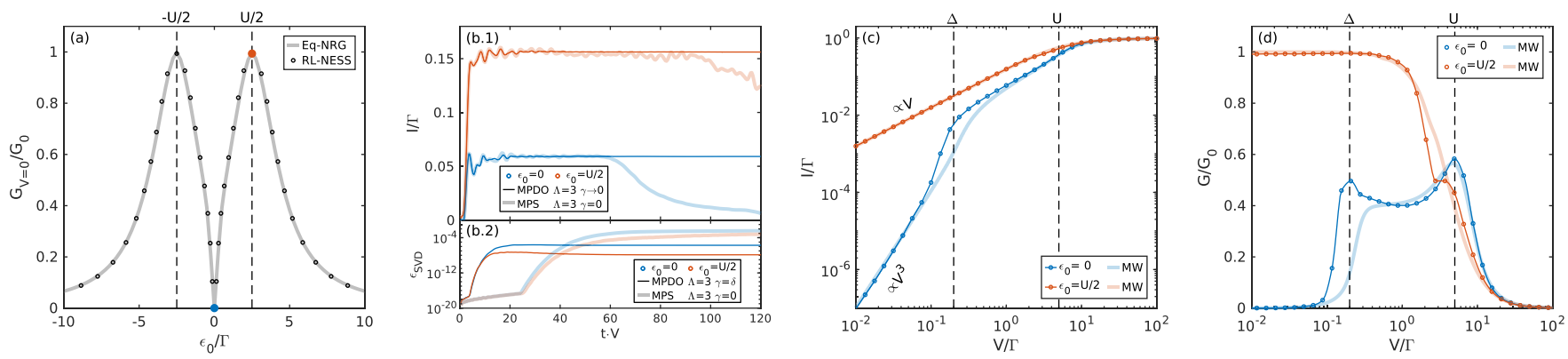

FIG. 5. RL-NESS simulation results for the interacting two-level model (I2LM) with level spacing $\Delta=\frac{\Gamma}{5}$ and interaction $U=5 \Gamma$, marked by dashed lines. The simulation is run with discretization parameters $\Lambda=3, \delta=0.1 D^{*}, D^{*}=\frac{V}{2}$, linearly extrapolated to $\gamma \rightarrow 0$ from $\gamma / \delta=4,2,1$, and with simulation parameters $\chi=256, R=64$. (a) Zero-bias linear conductance as a function of gate voltage $\varepsilon_{0}$, calculated by RL-NESS at $V=0.01 \Gamma$ (circles) and compared to the numerically exact equilibrium NRG result (shaded). (b.1) Comparison of the RLNESS MPDO evolution $(\chi=256$ ), after extrapolation to $\gamma \rightarrow 0$ (solid), and the NRG-tDMRG MPS evolution $(\chi=1024)$ for $\gamma=0$ (shaded), both with the same discretization, at $\varepsilon_{0}=0$ (blue) and $\frac{U}{2}$ (red), and bias voltage $V=\Gamma$. (b.2) Truncation error (sum of singular values squared accumulated over several time steps) plotted for the RL-NESS MPDO with $\gamma=\delta$ (solid) and the NRG-tDMRG MPS (shaded) evolution. (c) NESS current and (d) its derived differential conductance at finite bias, as calculated by RL-NESS for two gate voltages, corresponding to the valley at $\varepsilon_{0}=0$ (blue) and the peak at $\varepsilon_{0}=\frac{U}{2}$ (red). The low-bias behavior of the current exhibits a linear dependence for the peak, but a cubic dependence for the valley, where the linear response conductance thus vanishes quadratically in $V$. For comparison, the equilibrium spectral function of the I2LM is plugged into the Meir-Wingreen formula for the current and differential conductance (shaded). Note that it quantitatively captures the small- and large-bias features, but qualitatively misses various physical features in the intermediate bias regime.

given by

$$
\begin{aligned}
H_{\mathrm{dot}}^{\mathrm{ILM}} & =\varepsilon_{1} n_{1}+\varepsilon_{2} n_{2}+U n_{1} n_{2}, \\
\varepsilon_{1,2} & \equiv \varepsilon_{0}-\frac{1}{2} U \mp \frac{1}{2} \Delta .
\end{aligned}
$$

Both levels are coupled symmetrically to the left and right leads, so that the lead and coupling Hamiltonians are given by Eqs. (4) and (5), with equal hybridization $\Gamma_{\alpha \lambda}=\Gamma$. For simplicity, we take all the dot-lead couplings to be real and with the same sign. Since our main goal is demonstrating the method rather than studying the model, we do not explore the full impurity parameter space, but concentrate on restricted yet representative sets of parameter values. The level spacing and the interaction are fixed to $5 \Delta=\Gamma=\frac{U}{5}$ such that $\Delta<\Gamma<U$, thus having a separation of energy scales in a strongly correlated regime, and the bias and gate voltages are varied.

First, we explore the conductance in the small-bias linear response regime. Due to the Fermi-liquid nature of the lowenergy fixed point, the $T=0$ linear response conductance is determined by the total phase shift, which in turn is set by the Friedel sum rule $[48,49]$, leading to the relation

$$
G=G_{0} \sin ^{2}\left(\pi n_{d}\right),
$$

with $G_{0}=\frac{e^{2}}{h}$ the conductance quantum, and $n_{d}$ the total dot occupation in equilibrium, which can be calculated by equilibrium NRG. We show our results for $V=0.01 \Gamma \ll \Delta, \Gamma, U$ in Fig. 5(a) (circles) vs NRG (shaded). At gate voltage $\varepsilon_{0}=0$ the system is particle-hole symmetric, and the impurity is occupied exactly by one electron, such that the linear conductance vanishes. At $\varepsilon_{0} \approx \mp \frac{U}{2}$ the dot population is close to $1 \pm \frac{1}{2}$, respectively, hence the linear conductance features Coulomb blockade peaks with height $G_{0}$ (red dot).

Next, the NESS current is calculated at finite bias for two gate voltages $\varepsilon_{0}=0$ and $\frac{U}{2}$ corresponding, respectively, to the valley and the peak (in the zero-bias conductance).
The convergence to a steady state is now demonstrated for the interacting case in Fig. 5(b.1). Note again the decay of the oscillations and the saturation of the current, as opposed to the MPS calculation without dissipation $(\gamma=0)$, which eventually drifts off due to truncation error. This is clearly demonstrated in Fig. 5(b.2), in which the truncation error of the MPDO simulation saturates, while for the MPS simulation it continues to grow until the physical state is lost. The current for the full bias range is shown in Fig. 5(c). For $\varepsilon_{0}=\frac{U}{2}$ the low-bias behavior exhibits a linear dependence (shaded red), as expected. For $\varepsilon_{0}=0$, however, the linear response term vanishes by symmetry, and as the current is an odd function of the bias voltage, the next term is expected to be cubic in the bias voltage, as is indeed observed (shaded blue). In the limiting regime of very large bias, i.e., $V$ exceeding all other energy scales (except for bandwidth $D$ ), the current saturates for both cases to the maximal value of $\Gamma$, directly corresponding to the two conduction channels at coupling strength $\Gamma$ each. The differential conductance is shown in Fig. 5(d), with peaks corresponding to conductance channels opening up. For $\varepsilon_{0}=0$ we get very clear peaks, with the first conductance channel opening at $\Delta$ with sequential tunneling and thus fluctuations between the two dot levels, and the second conductance channel opening at $U$, corresponding to full charge fluctuations in the dot occupation. For $\varepsilon_{0}=\frac{U}{2}$, a single-particle level is midway between the two chemical potentials, so there is already a single channel fully open at zero bias, resulting in a differential conductance of $G_{0}$. The differential conductance starts dropping close to $V=\Gamma$ to about half its value. In the vicinity of $V=U$ there is a shoulder, beyond which the differential conductance drops to zero since the current saturates.

As an interesting comparison, consider an approximate form of the Meir-Wingreen formula for the steady-state current [50]. The exact version of this formula reads as for the 
I2LM

$$
I=\frac{i}{4 \pi} \int_{-\frac{V}{2}}^{\frac{V}{2}} d \omega \operatorname{tr}\left\{\Gamma(\omega)\left[G^{R}(\omega)-G^{A}(\omega)\right]\right\},
$$

where $G^{R, A}(\omega)$ are the exact retarded and advanced impurity nonequilibrium Green's functions $(2 \times 2$ matrices for the two impurity modes), and $\Gamma(\omega)=\Gamma\left(\begin{array}{ll}1 & 1 \\ 1 & 1\end{array}\right)$, corresponding to symmetric and equal hybridization of both modes to the two leads (for details see Appendix B). There is of course no known expression for the nonequilibrium Green's functions. However, one could calculate the equilibrium $(V=0)$ spectral function, e.g., by fdm-NRG [51], and plug it into Eq. (24). This approximation is valid in the linear response regime, and is expected to also produce quantitatively good results for large bias (with respect to all impurity energy scales), but in the intermediate regime is uncontrolled. Figure 5 therefore also shows the steady-state current (c) and differential conductance (d) obtained in this manner (shaded).

We see that the equilibrium spectral function results agree quantitatively with our nonequilibrium results in the low- and large-bias limits. Note that this agreement holds not only for the leading-order term (as one could prove), but also to the next order, e.g., the $\varepsilon_{0}=0$ cubic dependence. Interestingly, for $\varepsilon_{0}=0$ they also capture the charge-fluctuation peak at $V=U$ in the intermediate region, but only hint at the level fluctuation peak at $V=\Delta$. On the other hand, for $\varepsilon_{0}=\frac{U}{2}$, they completely miss the shoulder in the drop of the differential conductance. Thus, we conclude that the RL-NESS method successfully reproduces the current and differential conductance in the known limits, but also gives physically accurate results in the intermediate regime.

\section{DISCUSSION}

To conclude, in this work we have introduced RL-NESS, a numerically exact algorithm for finding the steady state of general impurities far from equilibrium. It builds on the power of equilibrium NRG in addressing equilibrium quantum impurities with widely separated bare and emergent energy scales, and brings it into the nonequilibrium realm. The method is based on coherently coupling the impurity to appropriately log-linearly discretized leads, which in turn are subject to weak Lindblad driving representing incoherent reservoirs. This model setup corresponds to the physical picture of, e.g., a quantum dot coherently coupled to quantum wires, which are in turn coupled to a classical voltage bias source. The resulting system is numerically simulated by a combination of NRG reduction of the high-energy degrees of freedom, followed by tDMRG-based MPDO Lindblad evolution. We benchmark our approach by presenting results for both noninteracting and interacting models. The accuracy of these demonstrate the power of our method, accompanied with a detailed analysis of all error sources and their treatment.

One can envision different ways to try to improve the algorithm. Having shown that an efficient representation of the steady state as a tensor network exists, it would be useful to search for more compact representations. One candidate for such a representation is the locally purified tensor-network ansatz $[40,44]$, which enforces physical constraints on the density operator such as positivity, and as such resides in a smaller manifold. However, it is not guaranteed that such an ansatz will efficiently capture the entanglement structure of the steady state [52], as preliminary investigation seems to suggest for the case at hand. So-called disentanglement schemes for the ancilla index [53] might improve the situation, but require further investigation. Recent works [54,55] claim that the entanglement structure of the chain geometry is not optimal, suggesting that applying time evolution in the star geometry might result in a slower growth of entanglement entropy, thus requiring a smaller bond dimension. Testing this idea together with RL-NESS is left for future work. Another direction which might be worth investigating is directly solving the Lindblad equation $\mathcal{L} \rho=0$ for the steady state [56,57], instead of obtaining it by real-time evolution.

It would be interesting to apply RL-NESS to more complicated models, such as the single-impurity Anderson model [2], the interacting resonant level model [58], and the I2LM with nonsymmetric coupling [59,60], all of which are expected to demonstrate Kondo-type physics. These models effectively have more lead channels, and thus require a larger RI low-energy subspace, i.e., larger $R$, which in turn requires an improvement of the algorithm or its numerical implementation. RL-NESS already incorporates a temperature for each lead, and so can immediately be employed for finite-temperature calculations, as well as calculating thermal conductance, by assigning a different temperature to each lead. We also plan to leverage the success of RLNESS in obtaining a stable steady-state solution, in order to extract dynamical properties, i.e., time correlators and spectral functions. In the longer run, we envision the treatment of far-from-equilibrium higher-dimensional correlated quantum systems, using, e.g., the dynamical mean field approach [61-63].

\section{ACKNOWLEDGMENTS}

We thank M. C. Bañuls, J. I. Cirac, J. Eisert, F. Schwarz, and A. Werner for helpful discussions. M.L. thanks F. Schwarz for making her code, developed for Ref. [30], available to him at the initial stages of this project. This joint work was supported by the German Israeli Foundation (Grant No. I-1259-303.10). In addition, A.W. was supported by the US Department of Energy, Office of Basic Energy Sciences (Contract No. DE-SC0012704). J.v.D. was supported by the Deutsche Forschungsgemeinschaft under Germany's Excellence Strategy Grant No. EXC-2111-390814868. M.G. acknowledges additional support by the Israel Science Foundation (Grant No. 227/15), the US-Israel Binational Science Foundation (Grant No. 2016224), and the Israel Ministry of Science and Technology (Contract No. 3-12419).

\section{APPENDIX A: LINEAR-LOGARITHMIC DISCRETIZATION}

As specified in Sec. II B, the intervals $I_{n}$ are chosen such that in the range $\left[-D^{*},+D^{*}\right]$ they are of size $\delta$ and far from this range they scale exponentially as $\sim \Lambda^{n}$. This choice of intervals is achieved by defining a function $f(x)$ for positive $x$, which is linear for $x<\frac{D^{*}}{\delta}$ and has a smooth transition to 
exponential $\sim \Lambda^{x}$ for large $|x|:$

$$
\begin{aligned}
& I_{n \geqslant 0}=[f(n+z), f(n+1+z)], \\
& f(x)=\left\{\begin{array}{cc}
\frac{\delta}{\log \Lambda} \sinh \left[\left(x-\frac{D^{*}}{\delta}\right) \log \Lambda\right]+D^{*}, & x>\frac{D^{*}}{\delta} \\
\delta x, & x<\frac{D^{*}}{\delta}
\end{array}\right.
\end{aligned}
$$

with $n$ running on all integers such that the full band is covered up to the cutoff $D$. The edge of the last interval is then manually fixed to be $D$. The parameter $z \in[0,1)$ is referred to as the $z$-shift parameter (in the NRG jargon), and can be used to shift the lead energy levels. Since different $z$ shifts result in different yet equivalent discretizations, it is common practice to average simulations using different $z$ shifts in order to reduce numerical artifacts due to the discretization [64], especially when calculating spectral functions. In this work, however, it was sufficient to use $z=0$. The intervals for negative energies are taken as a mirror image of the positive intervals. This guarantees particle-hole symmetry for any $z$, at the cost of the interval closest to 0 not necessarily being of size $\delta$. In each interval a representative energy level is selected, with its energy $\varepsilon_{n}$ chosen as the arithmetic mean of the interval boundaries in the linear sector (below $D^{*}$ ) and the geometric mean in the logarithmic sector (above $D^{*}$ ). The coupling Hamiltonian is then integrated over each interval in order to derive the appropriate coupling $v_{\alpha n \lambda}$ of the new lead level with the impurity $\lambda$ level:

$$
\begin{array}{r}
\varepsilon_{n}= \begin{cases}\frac{f(|n|+1+z)-f(|n|+z)}{\log [f(|n|+1+z) / f(|n|+z)]}, & \left|\varepsilon_{n}\right|>D^{*} \\
\frac{f(|n|+1+z)+f(|n|+z)}{2}, & \left|\varepsilon_{n}\right| \leqslant D^{*}\end{cases} \\
v_{\alpha n \lambda}=\sqrt{\frac{\Gamma_{\alpha \lambda}}{2 \pi D}[f(|n|+1+z)-f(|n|+z)] .}
\end{array}
$$

\section{APPENDIX B: EXACT SOLUTION OF THE CONTINUOUS NONINTERACTING CASE}

The exact solution for a quadratic continuous system can be calculated in the Keldysh formalism. For noninteracting leads, all the effects of the couplings to the leads on the impurity are encoded in the hybridization function, defined between the $\lambda$ th and $v$ th impurity levels $(\lambda, v \in\{1, \ldots, m\})$ for lead $\alpha \in\{L, R\}$ as

$$
\Gamma_{\alpha}^{\lambda v}(\omega)=\pi \sum_{n} v_{\alpha n \lambda}^{*} v_{\alpha n v} \delta\left(\varepsilon_{n}-\omega\right)
$$

where $v_{\alpha n \lambda}$ are the coupling constants between the $\lambda$ th impurity level and the $n$th energy level of lead $\alpha$ (in the star geometry). In the case of continuous leads, the sum over dense levels $\varepsilon_{n}$ is understood as an integral over the energies. The total hybridization function is then defined as a sum on the hybridization functions of all leads:

$$
\Gamma^{\lambda v}(\omega)=\sum_{\alpha} \Gamma_{\alpha}^{\lambda v}(\omega)
$$

For a quadratic dot Hamiltonian $H$, the retarded and advanced Green's functions of the dressed impurity are then given by

$$
\begin{aligned}
& G^{R}(\omega)=[\omega-H+i \Gamma(\omega)]^{-1}, \\
& G^{A}(\omega)=[\omega-H-i \Gamma(\omega)]^{-1},
\end{aligned}
$$

where $H$ and $\Gamma$ are understood here to be $m \times m$ matrices. The NESS current can be obtained by the Meir-Wingreen formula [50], which for equal hybridization functions $\Gamma_{L}(\omega)=$ $\Gamma_{R}(\omega)=\Gamma(\omega) / 2$ simplifies to

$$
\begin{aligned}
I=\frac{i}{4 \pi} \int & d \omega\left[f_{L}(\omega)-f_{R}(\omega)\right] \\
& \times \operatorname{tr}\left\{\Gamma(\omega)\left[G^{R}(\omega)-G^{A}(\omega)\right]\right\},
\end{aligned}
$$

where $f_{\alpha}(\omega)$ is the lead specific Fermi-Dirac distribution. The Keldysh Green's function equals

$$
G^{K}(\omega)=-2 i \sum_{\alpha}\left[1-2 f_{\alpha}(\omega)\right] G^{R}(\omega) \Gamma_{\alpha}(\omega) G^{A}(\omega),
$$

and the impurity single-particle density matrix can then be obtained by integrating over it:

$$
\begin{aligned}
\left\langle d_{\lambda}^{\dagger} d_{\nu}\right\rangle & =\frac{1}{2}\left(\delta_{\lambda \nu}-\left\langle\left[d_{\nu}, d_{\lambda}^{\dagger}\right]\right\rangle\right) \\
& =\frac{1}{2}\left(\delta_{\lambda \nu}-\frac{i}{2 \pi} \int d \omega G_{\nu \lambda}^{K}(\omega)\right) .
\end{aligned}
$$

Specifying a box hybridization function for the $\lambda$ th level with half-bandwidth $D$ :

$$
\Gamma^{\lambda \lambda}(\omega)=\Gamma^{\lambda \lambda} \Theta(D-|\omega|),
$$

and taking coupling constants $v_{\alpha n \lambda}=v$ which are lead, $n$ and $\lambda$ independent [so that all the elements of $\Gamma(\omega)$ are equal], as is indeed the case for both models under investigation in the continuum limit, Eq. (B4) simplifies to

$$
I=\frac{i}{4 \pi} \int_{-\frac{V}{2}}^{\frac{V}{2}} d \omega \operatorname{tr}\left\{\Gamma \times\left[G^{R}(\omega)-G^{A}(\omega)\right]\right\},
$$

which can then be evaluated for any desired bias voltage. The Keldysh Green's function in Eq. (B5) also simplifies to

$$
\begin{array}{r}
G^{K}(\omega)=-2 i\left(1-\sum_{\alpha} f_{\alpha}(\omega)\right) G^{R}(\omega) \Gamma(\omega) G^{A}(\omega) \\
=\left\{\begin{array}{cc}
+2 i G^{R}(\omega) \Gamma G^{A}(\omega) & -D<\omega<-\frac{V}{2}, \\
-2 i G^{R}(\omega) \Gamma G^{A}(\omega) & +\frac{V}{2}<\omega<+D, \\
0 & \text { else }
\end{array}\right.
\end{array}
$$

resulting in a simple integral for the single-particle density matrix.

\section{APPENDIX C: EXACT EVOLUTION OF THE DISCRETE NONINTERACTING CASE: THE LYAPUNOV EQUATION}

The single-particle single-time correlation matrix $P_{r s}(t) \equiv\left\langle c_{r}(t) c_{s}^{\dagger}(t)\right\rangle$ encodes all information regarding single-time observables of interest in this paper, e.g., the impurity current. Furthermore, for quadratic systems, this matrix encodes all information about the state of the system, so that finding $P(t)$ amounts to fully solving the system. In the case of a quadratic Lindblad equation (both in the Hamiltonian and the dissipative terms), the exact evolution, as well as the steady-state solution, can be reduced to a continuous Lyapunov equation for $P$. The key parts of this reduction are derived in this Appendix, following Ref. [32]. We start from the most general Lindblad equation for 

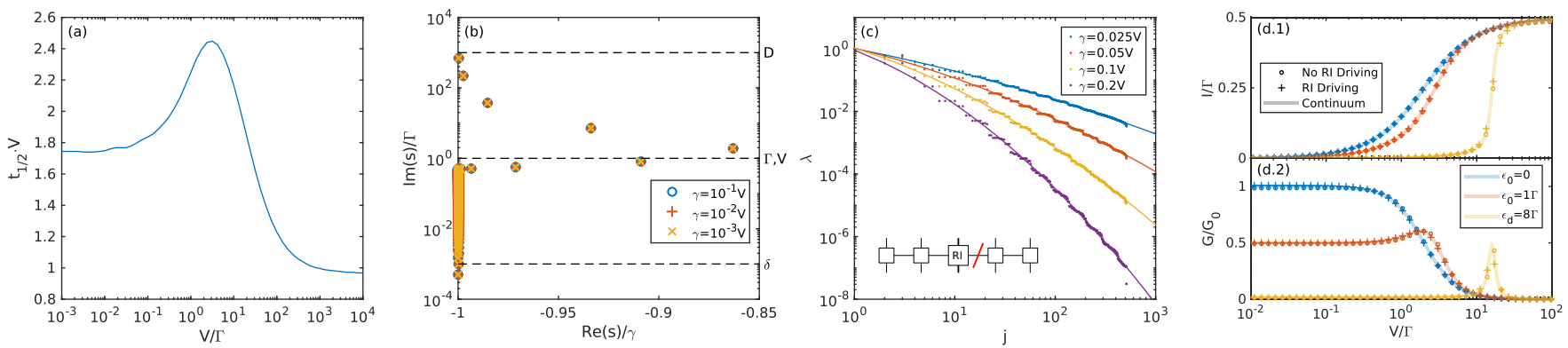

FIG. 6. (a) The time $t_{1 / 2}$ (multiplied by $V$ ) at which the current reaches half its final value, plotted for the RLM with $\varepsilon_{0}=0$ over a wide range of bias voltages. (b) The distribution in the complex plane of the eigenvalues of the matrix $A=-i H-\Lambda^{(1)}-\Lambda^{(2)}$, defined in Eq. (C3), for the RLM with several choices of driving rates $\gamma$. The imaginary parts, mostly corresponding to the Hamiltonian, are plotted in units of $\Gamma$, while the real parts, which are related to the Lindblad driving, are rescaled by $\gamma$. The closely bunched points near $\operatorname{Re}(\mathrm{s})=-\gamma$ correspond approximately to the single-particle energies of the Hamiltonian arising from the linear sector. (c) Example of the long-time limit steady-state singular value spectrum of the MPDO bond connecting the RI to one of the linear sector leads (as indicated by the red line in the cartoon). The spectrum was obtained for the RLM with $V=\Gamma, \delta=0.025 \mathrm{~V}, \chi=512, R=32$ and several values of $\gamma$. The singular values were rescaled such that the largest singular value for each $\gamma$ is 1 , and fitted to a log-Gaussian (solid line). (d) NESS current and differential conductance of the RLM as a function of bias voltage $V$ and at different gate voltages $\varepsilon_{0}$, as calculated with (pluses) and without (circles) Lindblad driving of the sites enclosed in the RI, and compared with the continuum limit (shaded). The current with and without the driving at the RI is calculated exactly for $\gamma / \delta=2,1$ (with $\delta=0.05 V$ ) and $\Lambda=8,6$, and is then linearly extrapolated to $\gamma \rightarrow 0, \Lambda \rightarrow 1$.

fermionic Lindblad operators $\left\{c_{q}\right\}$ :

$$
\begin{aligned}
\frac{\partial \rho}{\partial t}=-i[H, \rho] & +\sum_{m n} \Lambda_{m n}^{(1)}\left(2 c_{n} \rho c_{m}^{\dagger}-\left\{c_{m}^{\dagger} c_{n}, \rho\right\}\right) \\
& +\sum_{m n} \Lambda_{m n}^{(2)}\left(2 c_{m}^{\dagger} \rho c_{n}-\left\{c_{n} c_{m}^{\dagger}, \rho\right\}\right),
\end{aligned}
$$

where $\Lambda^{(1,2)}$ encode the Lindblad-driving rates. The time dependence of a general single-time observable $\langle A(t)\rangle \equiv$ $\operatorname{tr}(A \rho(t))$ is then given by

$$
\begin{aligned}
\frac{d\langle A\rangle}{d t}=-i\langle[A, H]\rangle & +\sum_{m n} \Lambda_{m n}^{(1)}\left\langle 2 c_{m}^{\dagger} A c_{n}-\left\{c_{m}^{\dagger} c_{n}, A\right\}\right\rangle \\
& +\sum_{m n} \Lambda_{m n}^{(2)}\left\langle 2 c_{n} A c_{m}^{\dagger}-\left\{c_{n} c_{m}^{\dagger}, A\right\}\right\rangle .
\end{aligned}
$$

Assuming a quadratic Hamiltonian $H=\sum_{m n} H_{m n} c_{m}^{\dagger} c_{n}$, and substituting $\langle A\rangle=P_{r s}$ into Eq. (C2), results in a differential continuous Lyapunov equation for $P$ :

$$
\begin{aligned}
\frac{d P}{d t} & =A P+P A^{\dagger}+M, \\
A & \equiv-i H-\Lambda^{(1)}-\Lambda^{(2)}, \quad M \equiv 2 \Lambda^{(1)} .
\end{aligned}
$$

The general solution of this equation, for some initial condition $P_{0}$, is

$$
P(t)=e^{A t} P_{0} e^{A^{\dagger} t}+\int_{0}^{t} e^{A t^{\prime}} M e^{A^{\dagger} t^{\prime}} d t^{\prime} .
$$

By diagonalizing $A$ (if possible) the integral can be explicitly calculated, resulting in a closed expression for $P(t)$. The steady-state solution is given by $P_{\text {NESS }}$ satisfying $\frac{d P_{\text {NESS }}}{d t}=0$. It can be obtained by solving the algebraic continuous Lyapunov equation

$$
A P_{\mathrm{NESS}}+P_{\mathrm{NESS}} A^{\dagger}+M=0 .
$$

By construction, $A$ has only eigenvalues with a nonpositive real part. If the sum of all pairs of eigenvalues of $A$ and $A^{\dagger}$ is nonzero, the equation is guaranteed to have a unique solution [65], i.e., a unique steady state, which can also be obtained by taking the infinite-time limit of Eq. (C4):

$$
P_{\mathrm{NESS}}=P(t \rightarrow \infty)=\int_{0}^{\infty} e^{A t^{\prime}} M e^{A^{t^{\prime}} t^{\prime}} d t^{\prime} .
$$

\section{APPENDIX D: EVOLUTION TIMESCALES}

In this Appendix we analyze the timescales of the current evolution for the RLM with $\varepsilon_{0}=0$, after discretization in the linear-logarithmic scheme, and with energy-independent Lindblad driving $\gamma$, as discussed in Sec. II B. The timescale of the initial rise in the current can be characterized by $t_{1 / 2}$, the time at which the current first reaches half of its final value. This timescale appears to be inversely proportional to the bias voltage $V$, as can be seen in Fig. 6(a) where $t_{1 / 2} V$ is of order unity over the full range of explored bias.

The timescale of the decay toward the steady state can be extracted for a quadratic model from the matrix $A$, defined in Eq. (C3). The (negative) real parts of the eigenvalues of this matrix dictate the decay rate of each mode. The ones with the smallest magnitude set a bound on the total decay rate of the system. For sufficiently small Lindblad driving, the imaginary part of the eigenvalues depends mainly on the Hamiltonian, while the real part will depend on the driving rates. Thus, for the RLM in the discussed discretization scheme, the real part of the eigenvalues naturally scales with $\gamma$, as can be seen in Fig. 6(b) for several choices of $\gamma$. Hence, the decay rate is proportional to $\gamma$, with the proportionality constant of order 1. Note that the fact that all eigenvalues have a finite (nonvanishing) negative real-part satisfies the criterion mentioned in Appendix $\mathrm{C}$ for a unique steady state.

\section{APPENDIX E: MPDO SINGULAR VALUE SPECTRUM}

In this Appendix we discuss the dependence of the longtime limit steady-state singular value spectrum of the MPDO on the Lindblad-driving rate $\gamma$. As an example we plot in 
Fig. 6(c) the singular value spectrum, taken at the bond connecting the RI to one of the linear sector leads (as indicated in the cartoon), for the RLM with parameters as given in the caption. First note that while the normalization of the wave function constrains the squared singular values of an MPS to sum up to 1 , the density operator normalization condition does not impose any constraint on the MPDO singular values. Thus, the global prefactor is arbitrary, and for clarity the singular values are rescaled such that the largest singular value for each $\gamma$ is 1 . As can be seen in the figure, the singular values decay at a faster than power-law rate, implying an efficient representation of the steady state as an MPDO with finite bond dimension $\chi$. Moreover, we observe that the decay rate grows monotonically with increasing $\gamma$, implying that larger $\gamma$ requires a smaller bond dimension in order to efficiently represent the state of the system.

A full characterization of the exact functional dependence of the singular values $\lambda_{j}$ on the index $j$ and the system parameters requires a more detailed analysis than carried out in this work. We do note, however, that we can fit it to a log-Gaussian behavior $\lambda_{j} \propto e^{-(a \log j+b)^{2}}$, with $a$ and $b$ the fitting parameters. We suspect that this specific behavior for an MPDO steady state is not coincidental since a similar behavior has been argued to occur for an MPS ground state [66]. We further observe that the fitting parameter $a$, which dictates the decay rate, is monotonic in $\gamma$ and goes to zero in the $\gamma \rightarrow 0$ limit. Thus, in this limit the required bond dimension $\chi$ diverges. This is to be expected, as in this limit the steady state corresponds to evolution to infinite time without dissipation, and we get the well-known exponential growth in entanglement entropy for unitary evolution.

\section{APPENDIX F: DRIVING RI SITES}

Figure 6(d) demonstrates that Lindblad driving of the RI itself has a negligible effect on the resulting current and differential conductance, with respect to an exact solution (which is attainable for the RLM). As argued in Sec. III, this is because the RI represents energy levels far above the voltage or temperature bias scales. These levels are not expected to be affected by the nonequilibrium conditions and thus only set the (renormalized) stage for the low-energy dynamics. Moreover, the exact solution of the modified Lindblad equation (without driving the RI) is still a valid approximation for the continuous system in the limits $\Lambda \rightarrow 1, \gamma=\delta \rightarrow 0$. This justifies turning off the driving for the interacting case, thus suppressing numerical artifacts arising due to the interplay between NRG and the dissipative dynamics.
[1] J. Kondo, Resistance minimum in dilute magnetic alloys, Prog. Theor. Phys. 32, 37 (1964).

[2] A. C. Hewson, The Kondo Problem to Heavy Fermions, Cambridge Studies in Magnetism (Cambridge University Press, Cambridge, 1993).

[3] K. G. Wilson, The renormalization group: Critical phenomena and the kondo problem, Rev. Mod. Phys. 47, 773 (1975).

[4] R. Bulla, T. A. Costi, and T. Pruschke, Numerical renormalization group method for quantum impurity systems, Rev. Mod. Phys. 80, 395 (2008).

[5] D. Goldhaber-Gordon, H. Shtrikman, D. Mahalu, D. AbuschMagder, U. Meirav, and M. A. Kastner, Kondo effect in a single-electron transistor, Nature (London) 391, 156 (1998).

[6] S. M. Cronenwett, T. H. Oosterkamp, and L. P. Kouwenhoven, A tunable kondo effect in quantum dots, Science 281, 540 (1998).

[7] J. Nygård, D. H. Cobden, and P. E. Lindelof, Kondo physics in carbon nanotubes, Nature (London) 408, 342 (2000).

[8] M. R. Buitelaar, A. Bachtold, T. Nussbaumer, M. Iqbal, and C. Schönenberger, Multiwall Carbon Nanotubes as Quantum Dots, Phys. Rev. Lett. 88, 156801 (2002).

[9] J. Park, A. N. Pasupathy, J. I. Goldsmith, C. Chang, Y. Yaish, J. R. Petta, M. Rinkoski, J. P. Sethna, H. D. Abruña, P. L. McEuen, and D. C. Ralph, Coulomb blockade and the kondo effect in single-atom transistors, Nature (London) 417, 722 (2002).

[10] W. Liang, M. P. Shores, M. Bockrath, J. R. Long, and H. Park, Kondo resonance in a single-molecule transistor, Nature (London) 417, 725 (2002).

[11] C. W. J. Beenakker, Theory of coulomb-blockade oscillations in the conductance of a quantum dot, Phys. Rev. B 44, 1646 (1991).
[12] A. Rosch, J. Paaske, J. Kroha, and P. Wölfle, Nonequilibrium Transport through a Kondo Dot in a Magnetic Field: Perturbation Theory and Poor Man's Scaling, Phys. Rev. Lett. 90, 076804 (2003).

[13] S. Kehrein, Scaling and Decoherence in the Nonequilibrium Kondo Model, Phys. Rev. Lett. 95, 056602 (2005).

[14] M. Pletyukhov and H. Schoeller, Nonequilibrium Kondo Model: Crossover from Weak to Strong Coupling, Phys. Rev. Lett. 108, 260601 (2012).

[15] S. Smirnov and M. Grifoni, Keldysh effective action theory for universal physics in spin- $\frac{1}{2}$ kondo dots, Phys. Rev. B 87, 121302(R) (2013).

[16] P. Mehta and N. Andrei, Nonequilibrium Transport in Quantum Impurity Models: The Bethe Ansatz for Open Systems, Phys. Rev. Lett. 96, 216802 (2006).

[17] A. B. Culver and N. Andrei, Nonperturbative approach to the nonequilibrium kondo model, arXiv:1912.02956.

[18] E. Boulat, H. Saleur, and P. Schmitteckert, Twofold Advance in the Theoretical Understanding of Far-From-Equilibrium Properties of Interacting Nanostructures, Phys. Rev. Lett. 101, 140601 (2008)

[19] L. G. G. V. Dias da Silva, F. Heidrich-Meisner, A. E. Feiguin, C. A. Büsser, G. B. Martins, E. V. Anda, and E. Dagotto, Transport properties and Kondo correlations in nanostructures: Time-dependent dmrg method applied to quantum dots coupled to wilson chains, Phys. Rev. B 78, 195317 (2008).

[20] J. Eckel, F. Heidrich-Meisner, S. G. Jakobs, M. Thorwart, M. Pletyukhov, and R. Egger, Comparative study of theoretical methods for non-equilibrium quantum transport, New J. Phys. 12, 043042 (2010)

[21] A. Dorda, M. Ganahl, H. G. Evertz, W. von der Linden, and E. Arrigoni, Auxiliary master equation approach within matrix 
product states: Spectral properties of the nonequilibrium anderson impurity model, Phys. Rev. B 92, 125145 (2015).

[22] M. Brenes, J. J. Mendoza-Arenas, A. Purkayastha, M. T. Mitchison, S. R. Clark, and J. Goold, Tensor-network method to simulate strongly interacting quantum thermal machines, Phys. Rev. X 10, 031040 (2020).

[23] G. Wójtowicz, J. E. Elenewski, M. M. Rams, and M. Zwolak, Open-system tensor networks and kramers' crossover for quantum transport, Phys. Rev. A 101, 050301(R) (2020).

[24] O. Hod, C. A. Rodríguez-Rosario, T. Zelovich, and T. Frauenheim, Driven liouville von neumann equation in lindblad form, J. Phys. Chem. A 120, 3278 (2016).

[25] Y. Tanimura and R. Kubo, Time evolution of a quantum system in contact with a nearly gaussian-markoffian noise bath, J. Phys. Soc. Jpn. 58, 101 (1989).

[26] Y. an Yan, F. Yang, Y. Liu, and J. Shao, Hierarchical approach based on stochastic decoupling to dissipative systems, Chem. Phys. Lett. 395, 216 (2004).

[27] H. Wang and M. Thoss, From coherent motion to localization: Dynamics of the spin-boson model at zero temperature, New J. Phys. 10, 115005 (2008).

[28] G. Cohen, E. Gull, D. R. Reichman, and A. J. Millis, Green's Functions from Real-Time Bold-Line Monte Carlo Calculations: Spectral Properties of the Nonequilibrium Anderson Impurity Model, Phys. Rev. Lett. 112, 146802 (2014).

[29] F. B. Anders, Steady-State Currents through Nanodevices: A Scattering-States Numerical Renormalization-Group Approach to Open Quantum Systems, Phys. Rev. Lett. 101, 066804 (2008).

[30] F. Schwarz, I. Weymann, J. von Delft, and A. Weichselbaum, Nonequilibrium Steady-State Transport in Quantum Impurity Models: A Thermofield and Quantum Quench Approach using Matrix Product States, Phys. Rev. Lett. 121, 137702 (2018).

[31] H. Breuer and F. Petruccione, The Theory of Open Quantum Systems (Oxford University Press, Oxford, 2007).

[32] F. Schwarz, M. Goldstein, A. Dorda, E. Arrigoni, A. Weichselbaum, and J. von Delft, Lindblad-driven discretized leads for nonequilibrium steady-state transport in quantum impurity models: Recovering the continuum limit, Phys. Rev. B 94, 155142 (2016).

[33] S. R. White, Density Matrix Formulation for Quantum Renormalization Groups, Phys. Rev. Lett. 69, 2863 (1992).

[34] U. Schollwöck, The density-matrix renormalization group in the age of matrix product states, Ann. Phys. 326, 96 (2011).

[35] A. Weichselbaum, Non-abelian symmetries in tensor networks: A quantum symmetry space approach, Ann. Phys. 327, 2972 (2012).

[36] A. Weichselbaum, Tensor networks and the numerical renormalization group, Phys. Rev. B 86, 245124 (2012).

[37] F. Güttge, F. B. Anders, U. Schollwöck, E. Eidelstein, and A. Schiller, Hybrid nrg-dmrg approach to real-time dynamics of quantum impurity systems, Phys. Rev. B 87, 115115 (2013).

[38] D. Gruss, K. A. Velizhanin, and M. Zwolak, Landauer's formula with finite-time relaxation: Kramers' crossover in electronic transport, Sci. Rep. 6, 24514 (2016).

[39] J. E. Elenewski, D. Gruss, and M. Zwolak, Communication: Master equations for electron transport: The limits of the markovian limit, J. Chem. Phys. 147, 151101 (2017).

[40] F. Verstraete, J. J. García-Ripoll, and J. I. Cirac, Matrix Product Density Operators: Simulation of Finite-Temperature and Dissipative Systems, Phys. Rev. Lett. 93, 207204 (2004).

[41] M. Zwolak and G. Vidal, Mixed-State Dynamics in OneDimensional Quantum Lattice Systems: A Time-Dependent Superoperator Renormalization Algorithm, Phys. Rev. Lett. 93, 207205 (2004).

[42] B. Buča and T. Prosen, A note on symmetry reductions of the lindblad equation: Transport in constrained open spin chains, New J. Phys. 14, 073007 (2012).

[43] V. V. Albert and L. Jiang, Symmetries and conserved quantities in lindblad master equations, Phys. Rev. A 89, 022118 (2014).

[44] A. H. Werner, D. Jaschke, P. Silvi, M. Kliesch, T. Calarco, J. Eisert, and S. Montangero, Positive Tensor Network Approach for Simulating Open Quantum Many-Body Systems, Phys. Rev. Lett. 116, 237201 (2016).

[45] K. Kraus, General state changes in quantum theory, Ann. Phys. 64, 311 (1971).

[46] M. A. Nielsen and I. L. Chuang, Quantum Computation and Quantum Information: 10th Anniversary Edition (Cambridge University Press, Cambridge, 2010).

[47] P. Corboz, R. Orús, B. Bauer, and G. Vidal, Simulation of strongly correlated fermions in two spatial dimensions with fermionic projected entangled-pair states, Phys. Rev. B 81, 165104 (2010).

[48] M. Goldstein and R. Berkovits, Interference effects in interacting quantum dots, New J. Phys. 9, 118 (2007).

[49] C. Karrasch, T. Hecht, A. Weichselbaum, J. von Delft, Y. Oreg, and V. Meden, Phase lapses in transmission through interacting two-level quantum dots, New J. Phys. 9, 123 (2007).

[50] Y. Meir and N. S. Wingreen, Landauer Formula for the Current through an Interacting Electron Region, Phys. Rev. Lett. 68, 2512 (1992).

[51] A. Weichselbaum and J. von Delft, Sum-Rule Conserving Spectral Functions from the Numerical Renormalization Group, Phys. Rev. Lett. 99, 076402 (2007).

[52] G. D. las Cuevas, N. Schuch, D. Pérez-García, and J. I. Cirac, Purifications of multipartite states: Limitations and constructive methods, New J. Phys. 15, 123021 (2013).

[53] J. Hauschild, E. Leviatan, J. H. Bardarson, E. Altman, M. P. Zaletel, and F. Pollmann, Finding purifications with minimal entanglement, Phys. Rev. B 98, 235163 (2018).

[54] C. Krumnow, J. Eisert, and O. Legeza, Towards overcoming the entanglement barrier when simulating long-time evolution, arXiv: 1904.11999.

[55] M. M. Rams and M. Zwolak, Breaking the Entanglement Barrier: Tensor Network Simulation of Quantum Transport, Phys. Rev. Lett. 124, 137701 (2020).

[56] J. Cui, J. I. Cirac, and M. C. Bañuls, Variational Matrix Product Operators for the Steady State of Dissipative Quantum Systems, Phys. Rev. Lett. 114, 220601 (2015).

[57] E. Mascarenhas, H. Flayac, and V. Savona, Matrix-productoperator approach to the nonequilibrium steady state of drivendissipative quantum arrays, Phys. Rev. A 92, 022116 (2015).

[58] A. O. Gogolin, A. A. Nersesyan, and A. M. Tsvelik, Bosonization and Sstrongly Correlated Systems (Cambridge University Press, Cambridge, 2004).

[59] C. Karrasch, T. Hecht, A. Weichselbaum, Y. Oreg, J. von Delft, and V. Meden, Mesoscopic to Universal Crossover of the Transmission Phase of Multilevel Quantum dots, Phys. Rev. Lett. 98 , 186802 (2007). 
[60] M. Goldstein, R. Berkovits, and Y. Gefen, Population Switching and Charge Sensing in Quantum Dots: A Case for a Quantum Phase Transition, Phys. Rev. Lett. 104, 226805 (2010).

[61] O. Sakai and Y. Kuramoto, Application of the numerical renormalization group method to the hubbard model in infinite dimensions, Solid State Commun. 89, 307 (1994).

[62] R. Bulla, A. C. Hewson, and T. Pruschke, Numerical renormalization group calculations for the self-energy of the impurity anderson model, J. Phys.: Condens. Matter 10, 8365 (1998).

[63] K. M. Stadler, Z. P. Yin, J. von Delft, G. Kotliar, and A. Weichselbaum, Dynamical Mean-Field Theory Plus
Numerical Renormalization-Group Study of Spin-Orbital Separation in a Three-Band Hund Metal, Phys. Rev. Lett. 115, 136401 (2015).

[64] W. C. Oliveira and L. N. Oliveira, Generalized numerical renormalization-group method to calculate the thermodynamical properties of impurities in metals, Phys. Rev. B 49, 11986 (1994).

[65] R. H. Bartels and G. W. Stewart, Solution of the matrix equation $\mathrm{AX}+\mathrm{XB}=\mathrm{C}[\mathrm{F} 4]$, Commun. ACM 15, 820 (1972).

[66] P. Calabrese and A. Lefevre, Entanglement spectrum in onedimensional systems, Phys. Rev. A 78, 032329 (2008). 